Revista de Psicología de la PUCP. Vol. XIV. No 2. 1996

\title{
LAS CINCO GRANDES DIMENSIONES DE LA PERSONALIDAD
}

\author{
Jan ter Laak' \\ Universidad de Utrecht
}

Este artículo revisa las distintas posiciones teóricas sobre las cinco grandes dimensiones de la personalidad, mostrando las semejanzas y diferencias entre las posturas teóricas. Esta contribución presenta lo siguiente: (a) la génesis del contenido y la estructura de las cinco dimensiones; (b) la fortaleza de las cinco dimensiones; (c) la relación de las cinco grandes dimensiones con otros constructos de personalidad; (d) discute el valor predictivo de las puntuaciones del perfil de las cinco dimensiones para criterios pertinentes; (e) analiza el estatus teórico de las cinco dimensiones; ( $f$ )discute críticas históricas sobre las cinco grandes dimensiones y se formulan respuestas a estas críticas; ( $\mathrm{g}$ ) hace conjeturas para el futuro de las cinco grandes dimensiones; y $(h)$ concluye con algunas conclusiones y comentarios.

Palabras Claves: cinco grandes dimensiones en personalidad, personalidad, diferencias individuales, adultos.

The Big Five dimensions of individual differences in personality

This is a review article about the different theoretical positions on the Big Five dimensions of personality .This article presents the similarities and differences among the theoretical positions following a thorough reflection on the following topics: (a) the genesis of the content and structure of the Big Five; (b) the robustness of the five dimensions; (c) a description of the research on the relationship of the Big Five with other personality constructs; (d) a discussion about the predictive value of the Big Five profile scores for relevant criteria; (c) the theoretical status of the Five is analyzed; (f) historical criticisms on the Big Five are discussed; $(g)$ some guesses for the future of the Five are put forward; and $(h)$ conclusions and remarks about the Five dimensions of personality are drawn and made.

Keywords: Big Five, personality, individual differences, adults.

1 Profesor asociado de la1 Universidad de Utrecht, Departamento de Psicología del Desarrollo. Heidelberglaan 2, 3508 TC Utrecht, Los Países Bajos. Correo electrónico: TERLAAK@FSW.RUU.NL 



\section{Introducción}

Las personas son en algunos aspectos iguales, en otros diferentes y en otros, únicos (Kluckhohn y Murray, 1953). La personalidad puede describirse como un conjunto de componentes que forman un sistema. Mayer (1995) distinguió cerca de 400 componentes a saber: intelecto, carácter, temperamento, disposición, humor, actitudes, tendencias conductuales, rasgos, estados, competencia y funciones mentales. Estos componentes pueden describirse verbalmente (en el lenguaje cotidiano o científico), metafóricamente (por ejemplo, el ego es como un jinete a caballo) o matemáticamente (por ejemplo, un factor en el análisis factorial).

Los rasgos o las disposiciones son un conjunto de componentes para describir diferencias individuales. Estos han conquistado un lugar central en la psicología. A partir de la obra de Galton Inquiry into human faculties and its development (1883). Thomae (1990) ha demostrado con datos bibliométricos que los rasgos y las disposiciones son centrales en la psicología de la personalidad europea y (norte) americana. Todos los rasgos no son completamente diferentes; es decir, independientes uno de otro. Los análisis correlacionales de los instrumentos que miden los rasgos o disposiciones regularmente indicaban dos dimensiones, interpretadas como extroversión y neuroticismo. Estos conceptos nos recuerdan a Jung y Freud y fueron llamados las Dos Grandes siendo Wiggins (1968) el que probablemente utilizó primero esta expresión. La abundante investigación correlacional en el enfoque psicoléxico que uriliza adjetivos de personalidad agregó tres dimensiones: agradabilidad, escrupulosidad y apertura. Éstas 
fueron llamadas las Cinco Grandes Dimensiones (ver por ejemplo, Goldberg, 1981). Como resultado, las diferencias individuales en la personalidad son retratadas en cinco dimensiones. Así una persona específica puede ser descrita con un perfil de puntajes en cinco dimensiones.

La investigación de las cinco grandes dimensiones se ha incrementado últimamente, lo que se aprecia en los simposios sobre este tema, ediciones especiales como la del European Journal of Personality Psychology (De Raad \& Van Heck, 1994), y a través de un activo grupo de investigadores internacionales. Inclusive el crítico de las cinco grandes dimensiones, McAdams (1994a), en su manual, organiza la teoría disposicional o de los rasgos bajo Los Cinco Rasgos Básicos. Hofstee (1994, p. 152) llama a la investigación de las cinco grandes dimensiones un "paradigma" que lo iguala con la tradición psicométrica (analítico factorial) de investigación en personalidad. La historia de las cinco grandes dimensiones se describe repetidamente. Presumiblemente, es ahora el momento, que uno puede mirar hacia atrás los esfuerzos que llevaron al descubrimiento de las dimensiones esenciales de la personalidad (por ejemplo, John \& Angleitner, 1988; John, 1990; Mount \& Barrick, 1991; John \& Robins, 1993; Hofstee, 1994; De Raad, en prensa 1996). Como Wolfe (1993, p. 284) escribe: "Por fin, estamos comenzando a llegar a algún lugar". Las dimensiones se derivan del lenguaje cotidiano; la persona común puede formarse un cuadro de las dimensiones y puede fácilmente conectarse a la conducta; así las personas pueden describirse a sí mismas y a los otros. Además un acuerdo sobre la taxonomía de las dimensiones es útil en la investigación, por lo menos como una norma para comparar los muchos conceptos y escalas de personalidad (Snow, 1973; MacKay 1993).

Esta contribución contiene, en primer, lugar el origen del contenido y la estructura de las cinco dimensiones. En segundo lugar presenta la fortaleza de las cinco dimensiones, es decir, se investiga la generabilidad de sus métodos, categorías lingüísticas, culturas, lenguas, y edad. Tercero, se describe la relación de las cinco grandes dimensiones con otros constructos de personalidad. Cuarto, se discute el valor predictivo de los puntajes del perfil de las cinco dimensiones para criterios pertinentes. Quinto, se analiza el status teórico de las cinco dimensiones. Sexto, se discuten críticas históricas sobre las cinco grandes dimensiones y además, se formulan algunas 
respuestas y defensas a estas críticas y se hacen algunas conjeturas para el futuro. Por último, se sacan algunas conclusiones y comentarios.

\section{Génesis del Contenido y de la Estructura de las Cinco Grandes Dimensiones}

Aunque el laboratorio experimental de Wundt es onsiderado a menudo como el comienzo de la psicología científica, es más verosímil que la psicología comenzara como una ciencia empírica al considerar las diferencias individuales en las capacidades entre las personas. Galton fue el primero en preparar el terreno de los instrumentos para evaluar diferencias en la inteligencia. En 1884, él escribió un artículo sobre la Medición del carácter. El léxico y el uso del diccionario como un medio para definir el contenido de la personalidad ya era recomendado por el psicólogo alemán Rumelin (1890). Señaló que los conceptos que primero vienen a la mente para expresar observaciones de personas eran aquellos conceptos, ... "welche die Sprache durch ein besonderes Wort ..." [que el lenguaje expresa por medio de una palabra especial] que es capaz de expresar. Este es un enfoque ampliado de las diferencias individuales. Uno tiene que seleccionar palabras de todo el diccionario y establecer un consenso sobre las palabras de persona(lidad). Esto fue el comienzo del enfoque psicoléxico de la personalidad. Klages (1926/1932) y Baumgarten (1933) también se refirieron al lenguaje natural como una fuente para la construcción de una taxonomía descriptiva de las diferencias individuales.

Allport, formado en Heidelberg (Alemania), y Odbert (1936) fueron los primeros que realizaron un estudio psicoléxico completo. Extrajeron términos pertinentes a la personalidad incluidos en el diccionario Oxford, asumiendo que la mayoría de las características sociales de personalidad relevantes y sobresalientes habían sido codificadas en el lenguaje natural, seleccionando 17953 términos. Cattell (1943) utilizó esta lista para los 16 Factores de Personalidad (16PF), enfoque que revivió en los ochenta. Goldberg (1981) lo describe como sigue: "Aquellas diferencias individuales que son más significativas en las transacciones diarias de las personas entre sí serán finalmente codificadas en su lenguaje. Mientras más importante sea la diferencia, más personas la notarán y desearan hablar de ello, teniendo como resultado que al fin inventarán una palabra para ello" (pp. 141- 
142). La gran cantidad de palabras referidas a la persona les causó problemas a Allport y Odbert quienes requirieron de criterios de selección. Los rasgos de personalidad eran considerados tendencias determinantes personalizadas y generalizables determinantes y modos estables y consistentes del ajuste del individuo a su ambiente. Fueron excluidos los estados, las características físicas y los juicios evaluativos (por ejemplo, acerca de la reputación de una persona). Además, los autores no estaban interesados en la visión nomotética ortodoxa (sensaciones, habilidades, necesidades, actitudes, instintos y sentimientos), en la visión nomotética dinámica (ego, culpa, sentimientos, agresión, represión) ni en los rasgos específicos. Aunque con el uso de estos criterios y preferencias, la selección de rasgos léxicos no era simple, pero finalmente se logrará obtener un número más reducido y manejable. Cattell (1943) utilizó la lista de Allport \& Odbert con criterios no circunscritos claramente(John y Angleitner, 1988) de donde seleccionó 35 escalas bipolares. El análisis factorial de las escalas produjeron 12 factores. Para completarlo, añadió cuatro escalas más: quedando conformado los 16 PF. Fiske (1949) utilizó 22 de las escalas de Cattell y el análisis factorial dio como resultado cinco factores. El informe de Fiske no consiguió mucha atención, en parte porque él mismo no estaba muy entusiasmado con este enfoque lingüístico para la conducta (Goldberg, 1995).

En 1961, dos psicólogos norteamericanos del ejército escribieron un informe técnico para dicha institución (reimpreso en 1992) donde utilizaron 35 rasgos "representativos" para el campo de la personalidad. Los rasgos fueron puntuados por ocho muestras, que se conocían mutuamente de tres días a un año. Extrajeron e interpretaron cinco factores: I Surgencia II Agradabilidad III Seguridad IV Estabilidad Emocional y V Cultura. Éstos se consideran los primeros nombres para las cinco grandes dimensiones. Los autores estaban impresionados por las semejanzas: "Diferencias en las muestras, los calificadores, la dirección y tipo de familiaridad tuvieron poco efecto sobre la estructura factorial que subyace a las calificaciones de los rasgos de personalidad" (p. 244). Aunque fueron cautos sobre el significado: "Es improbable que los cinco factores identificados sean los únicos factores de personalidad fundamentales", éste fue el comienzo de las cinco grandes dimensiones. 
En 1963, Norman añadió 171 términos a la lista de Allport y Odbert. y excluyó los términos oscuros y evaluativos, así como palabras que se refierían a la apariencia anatómica y física. Se incluyó rasgos, estados internos, roles sociales y afectos. Los análisis produjeron dimensiones, pero Norman dudó de la realidad psicológica de estas dimensiones lingüísticas, porque era el "Zeitgeist" de las décadas del sesenta y del setenta (Passini \& Norman, 1966).

El resurgimiento contemporáneo se debe probablemente a Goldberg (1981). Utilizó 1710 adjetivos, 1431 de los cuales fueron extraídos de Norman; organizó los términos en 75 categorías de similitud y extrajo 13 factores. Los primeros cuatro fueron fácilmente interpretables, el quinto era una combinación de componentes sociales del funcionamiento mental y experiencial. Digman, enseñando estadística, utilizó los puntajes de los rasgos para explicar el análisis multivariado y se decidió por cinco factores (Digman \& Takemoto-Chok, 1981).

Peabody (1987) utilizó un método intencional, seleccionó rasgos representativos con la ayuda de una clasificación racional y encontró soporte para las cinco dimensiones. Goldberg no construyó el instrumento que se usa mayormente. Es un hecho histórico importante, que este instrumento, el NEO PI fue construido por Costa y McCrae (1985) quienes no tomaron como su punto de partida la lista completa de rérminos de personalidad, sino los 16PF de Cattell. Como se mencionó, Cattell utilizó 35 escalas, extrajo 12 y ańadió 4 factores. La disponibilidad de un instrumento de diferencias individuales, una prueba simple, estimula la investigación (correlacional). Esto ocurrió mucho con el NEO PI como lo demuestra la siguiente sección.

Este camino sinuoso, a través de la historia, desde Galton a Goldberg condujo a nombres ligeramente diferentes para los cinco factores. Recientemente, los factores son acompañados por seis facetas, que representa un nivel jerárquico inferior al de las dimensiones. Los factores y las facetas son considerados por algunos adherentes a las cinco grandes dimensiones como los elementos básicos de personalidad. Los factores (I al V, ver tabla 1) son ordenados de acuerdo a la varianza que ellos explican. Como consecuencia una persona es un patrón de puntajes de los cinco factores. 
El anagrama $\mathrm{OCEAN}^{2}$ ayuda a memorizarlos, sugiriendo para bien o para mal la profundidad insondable o la superficie turbulenta de las dimensiones de personalidad.

Tabla 1. Dimensiones léxicamente derivadas de personalidad: Las cinco grandes dimensiones y facetas.

I Extroversión (Surgencia): afecto, gregarismo, asertividad, actividad, búsqueda de emociones, emociones positivas.

II Agradabilidad (Simpatía): confianza, honradez, altruismo, cumplimiento, modestia, sensibilidad.

III Consciencia (Conformidad, Seguridad): competencia, orden, obediencia, lucha por el logro, autodisciplina, reflexión.

IV Neuroticismo (Estabilidad Emocional, Inquietud): Ansiedad, hostilidad colérica, depresión, timidez, impulsividad, vulnerabilidad.

V Apertura a la Experiencia (Cultura, Intelecto, “Intelectancia”): fantasía, estética, sentimientos, acciones, ideas, valores.

El enfoque léxico suministra el contenido del lenguaje de la persona(lidad); es decir, las unidades. La relación entre las unidades, esto es, su estructura se modela desde Cattell (1943) por medio del análisis factorial, principalmente utilizó la técnica de reducción de datos. Además, el análisis factorial o mayormente el análisis de los componentes principales con rotación varimax implica que uno acepta que la "estructura simple" es el retrato adecuado de las dimensiones de la personalidad. Una persona puede ser caracterizada por puntajes en las cinco dimensiones independientes. La estructura simple se describe en los Vectores de la Mente de Thurstone (1935), quien utilizó esta técnica para el análisis de 56 pruebas de inteligencia (Thurstone \& Thurstone, 1941). Este estuvo muy cerca de "descubrir" las dimensiones de la personalidad, porque empezó a medir dimen-

2 Este anagrama proviene de las iniciales de los factores en Inglés: Openness, Conscience, Extroversion, Agreeableness y Neuroticism. 
siones de personalidad, especialmente la neurosis (Thurstone $\&$ Thurstone, 1930). Sin embargo, su nombre está conectado a siete Habilidades Mentales Primarias (PMA) y no a las cinco (o ¿̨hubieran sido siete?) dimensiones de la personalidad.

Wiggins (1979) ha utilizado el modelo circunflejo para la descripción del campo de los rasgos interpersonales. Ocho sagmentos del modelo representan diferencias interpersonales: amor y estatus, reacciones emocionales, roles y status, características, pensamientos y percepciones. Utilizando el diseño de la faceta de Foa (1965), produjo ocho grupos por tres dicotomías jerárquicas: Aceptar-Rechazar; Uno mismo-El otro y AmorEstatus.

El amor y el estatus también son llamados Crianza y Dominancia. El modelo circunflejo basado en un enfoque tipo faceta permite predecir correlaciones: $1.00,-1.00, .50$ y -.50 entre las características. En el modelo completo se tienen las siguientes relaciones: Ambicioso-Dominante vs. Flojo-Sumiso (-1.00); Frío-Calculador vs. Cálido-Agradable $(-1.00)$ y Arrogante-Calculador vs. Frío-Peleador $(+.50)$ y Reservado-Introvertido vs. Flojo-Sumiso (-.50). Si estas relaciones se encuentran en la investigación empírica, se dice que el modelo contiene "fidelidad estructural". Este concepto es interesante porque es una extensión significativa del concepto de confrabilidad. No se refiere a la confiabilidad de (suma) de puntajes únicos en pruebas, sino al orden de un conjunto de puntajes (Loevinger, 1957). El diseño de Wiggins tiene espacio sólo para dos factores, mientras que las cinco grandes dimensiones están referidas a los cinco factores. De Raad \& Hofstee (1993) integraron los dos modelos al construir 10 modelos circunflejos de las diez combinaciones $2 \times 2$ de las cinco dimensiones. Esta combinación $2 \times 2$ era razonable porque los adjetivos a menudo cargaban sustancialmente en los dos factores. Los ejemplos de los grupos de adjetivos de personalidad de acuerdo a este modelo son : I+II- (dominante, dominador, poderoso) y I-II+ (tímido, pasivo, sumiso). Si el evaluador quiere dar puntaje a una persona en los cinco factores ortogonales, una regla empírica derivada explica las cargas en los cinco factores separadamente (Hendricks, Hofstee, De Raad \& Angleitner, 1995). Para dar un ejemplo, el factor Y (Extroversión) el cual da ciertamente el mayor peso a la escala 1 (Extroversión: +1.19), pero también da cierto peso a la escala 2 (Agradabilidad: 
-.07), a la escala 3 (Escrupulosidad: +.05), a la escala 4 (Neuroticismo: .20) y a la escala 5 (Apertura a la experiencia: -.28).

La estructura de los estudios de dimensiones se impone por el análisis factorial (exploratorio) y por el análisis del componente principal. Estas técnicas imponen la "estructura simple" y conducen a una cantidad de factores independientes. Recientemente, la estructura simple y el modelo circunflejo se integran. El retrato de una persona es un perfil de 5 puntajes en 5 dimensiones independientes.

\section{La Fortaleza de las Cinco Dimensiones: generalizabilidad sobre los métodos, las categorías lingüísticas y las edades}

Campbell y Fiske (1959) presentaron la matriz multimétodo multirasgo para investigar la validez del constructo en las pruebas. Una prueba tiene que ser insensible a las diferencias de métodos y tiene que converger con constructos similares y diverger de constructos diferentes. Una dimensión de personalidad es un constructo válido si atrae una substancial varianza independientemente del método de medición y si mantiene su significado entre los otros constructos. La fortaleza de las cinco dimensiones puede investigarse de la misma manera. ¿Aparecen el mismo número y las mismas dimensiones utilizando diferentes métodos, como por ejemplo, los informes, descripciones libres; los adjetivos u oraciones cortas, las formas abreviadas o completas, y en diferentes edades? Además, si las cinco dimensiones son elementos básicos de personalidad, se tiene que encontrar la comparación transcultural. Recientes estudios representativos que pertenecen a esta pregunta se describen a continuación.

Los adjetivos de personalidad pueden completarse en oraciones: $\mathrm{x}$ es una (adejtivo) persona. Se les pide a los sujetos estimar hasta que punto los adjetivos se aplican a ellos mismos y/o a los demás. Hendricks, Hofstee \& Angleitner (1995) utilizaron oraciones cortas en lugar de adjetivos. Los sujetos se sintieron más cómodos al dar puntaje de este modo, algunos ejemplos son: "Se toma su tiempo para conversar; Se amarga rápidamente, Actúa sin planificar, Se siente desesperado". Este método produce la misma estructura que los adjetivos. 
De Raad (1992) investigó si las cinco dimensiones se encontraban también en los sustantivos y los verbos. En lo que se refiere a los sustantivos se utilizaron 755 . Una solución de cuatro factores reproduce cuatro de las cinco dimensiones: Intelecto/Apertura, II Extroversión, III Escrupulosidad y IV Agradabilidad. La Extroversión se mezcló con la Estabilidad Emocional (Neuroticismo). La estructura verbal se desvió de la estructura dimensional usual. Los verbos probablemente necesitan más especificación para ser útiles en inferencias disposicionales.

La investigación psicoléxica con adjetivos produjo consistentemente cuatro factores interpretables. El quinto factor era y es ambiguo (Cristal y Tupes, 1961/1992; De Raad, 1994). Los nombres provisionales para el quinto factor son: Cultura, Intelectualidad, Inteligencia, Apertura a la Experiencia. No se trata de la Competencia o la Inteligencia tal como se miden con las pruebas de CI. Las correlaciones entre los puntajes de las pruebas de inteligencia y este quinto factor son modestas $(r=.20-.30)$

Antes que los investigadores estuvieran de acuerdo con las cinco dimensiones, algunas veces se reportaron más dimensiones. Por ejemplo, un estudio psicoléxico holandés (Broken \& Smid, 1984) añadió dimensiones: Agresión, Progresividad, Animo depresivo, Caprichosidad y Superficialidad. Las últimas tres fueron descubiertas al volver a analizar las otras dimensiones. Por ejemplo, la escala de Animo Depresivo podía considerarse como una combinación lineal de Introversión y Caprichosidad. Estos autores mostraron que era posible refinar adicionalmente, mientras preserva más o menos las dimensiones originales.

Recientemente, ningún nuevo factor se ha añadido, pero dentro de cada dimensión se distinguen seis facetas (Ostendorf \& Angleitner, 1993). Saucier (1994) ha utilizado los 100 marcadores del modelo circunflejo integrado con los cinco factores para construir una versión de 40 ítemes. Este "Mini-Marcador" contenía las cinco dimensiones. Aparte de las consecuencias de la reducción de la prueba en la confiabilidad, los 40 items hicieron impresionantemente bien su trabajo.

Havill, Halverson \& Kohnstman (1994) y Kohnstman, Halverson, Havill \& Mervielde (1994) utilizaron las descripciones libres de los padres y maestros de la personalidad de los hijos y alumnos entre los 3 y los 12 
años ("Por favor, háblenos sobre su hijo, su alumno"). Las descripciones libres fueron categorizadas en 15 grupos. Las primeras cinco correspondieron a las cinco grandes dimensiones y del $70 \%$ al $80 \%$ de las descripciones podían atribuírselas a estas dimensiones. Las dimensiones no diferían en las muestras de los niños norteamericanos, holandeses y belgas. Las 10 categorías que quedaron eran específicas y no encajaban dentro de las cinco dimensiones, por ejemplo, "Maduro para su edad, Enfermedades, Ritmicidad, Conducta apropiada para su sexo, Amoroso/Pegajoso y Desempeńo escolar". Las cinco dimensiones atraen aparentemente muchas descripciones libres".

¿Se pueden aplicar las cinco dimensiones a niños, a otras edades, a los? Van Lieshout y Haselager (1992) utilizaron la adaptación holandesa del conjunto California C (Block 6 Block, 1980) e investigaron si sus datos de conjunto $\mathrm{C}$ de 2634 niños de tres a siete años de edad contenían las cinco dimensiones. El análisis de los componentes principales de los juicios de los padres y maestros mostró que muchos ítemes se saturaban en el componente Agradabilidad. La Estabilidad Emocional estuvo presente y las dimensiones del conjunto $\mathrm{C}$, Orientación de Logro y Dinámica se consideraron como facetas de la Formalidad y la Extroversión.

Los autores hicieron esfuerzos para encontrar semejanza del conjunto $\mathrm{C}$ (CCQ de Block) con las cinco grandes dimensiones, aunque también habían diferencias. John, Caspi, Robins, Moffit \& Stouthamer-Loeber (1994) utilizaban como Van Lieshout et al. (1992) datos CCQ de 350 niños étnicamente diferentes entre los 12 y los 13 años de edad; 48 de los 100 ítemes CCQ podían categorizarse en las cinco dimensiones (las "cinco grandes dimensiones CCQ"). Los ítemes eran suficiente e internamente consistentes, excepto los 7 ítemes en la dimensión Apertura (alfa de Cronbach de .53). Estos ítemes CCQ fueron utilizados como un índice para las cinco grandes dimensiones en los adolescentes varones. El análisis del componente principal en estas "cinco grandes dimensiones "de los ítemes CCQ ofrecieron dos dimensiones adicionales: VI Irritabilidad y VII Actividad Positiva. Existe algo común entre los adultos y los adolescentes varones, pero también hay una diferencia. El autor interpreta esto como un efecto específico de la edad. Se esperaba que las dos dimensiones adicionales desaparecieran cuando los adolescentes crecieran. 
Esta sección muestra que las cinco grandes dimensiones son consideradas como relativamente insensibles a diferentes métodos. Los autores parecen enfatizar la invarianza del método y las diferencias que estuvieron presentes no se discutieron.

\section{La validez de constructo de las cinco grandes dimensiones}

Las cinco grandes dimensiones son deliberadamente generales; es decir, se dirigen hasta cierto punto a ser "transituacionales" y "transcontextuales". Esto implica correlaciones substanciales con otras escalas generales de personalidad y correlaciones más bajas con constructos más específicos. Si algo se gana con las cinco dimensiones, entonces, sus correlaciones con todas las demás escalas deben ser más altas que las correlaciones de estas escalas mutuamente. La comparación de escalas en la investigación de validación de constructos se está llevando a cabo para lograr significado a través de los coeficientes de validez convergente y divergente.

Presley \& Martin (1994) compararon una escala de temperamento (TABC) con las cinco grandes dimensiones en 2,176 nińos preescolares. Padres y maestros juzgaban estas características en los niños. Los autores analizaron factorialmente la matriz correlacional (como estimados de las comunalidades en la diagonal de dicha matriz correlacional, utilizando correlaciones múltiples al cuadrado) y aplicando la rotación varimax. Esto produjo 23 factores con valores más grandes que 1.0. La prueba scree y otros criterios de contenido indicaron una solución de cinco factores: I Inhibición social, II Emocionalidad negativa, III Adaptabilidad, IV Nivel de actividad y V Persistencia en la tarea. El análisis factorial del formato maestro produjo tres factores: I Persistencia en la tarea, II Actividad inhibida Y III Emocionalidad Negativa. Los padres y los maestros no muestran la misma estructura. Los autores se impresionaron por la estructura a lo largo de todo el rango de edad. Al comparar los resultados del $\mathrm{TABC}$ con las cinco grandes dimensiones resaltaron: "Existe una similitud conceptual interesante entre los factores obtenidos en este estudio y estos factores en los adultos" (p. 433). Según Presley \& Martin se asemejan a la Introversion-Extroversión; La Adaptabilidad contiene facetas de Agradabilidad; Persistencia en la tarea va junto con Escrupulosidad; y Emocionalidad Negativa con Neuroticismo. La Apertura no estuvo pre- 
sente en los juicios de temperamento. Estos autores querían encontrar similitudes, pero también habían diferencias.

McCrae, Costa \& Piedmont (1993) compararon el Inventario Psicológico California (CP, Gough, 1987) y las cinco grandes dimensiones. Gough afirma que los ítemes en el CPI están cerca al lenguaje cotidiano. Esto nos recuerda al enfoque léxico. La comparación fue llevada a cabo por seis jueces que categorizaron los 480 ítemes en las cinco dimensiones. Las calificaciones de los seis pares de juicio en los ítemes variaron de .23 a .74. Se hallaron las correlaciones promedio más altas para Extroversión y Escrupulosidad (.65 y .65). Mientras que Apertura y Agradabilidad obtuvieron valores más bajos (.42 y .44). Los autores concluyeron que existen: “ ... correspondencias interpretables entre los constructos medidos por las escalas CPI y el contenido del ítem en las cinco dimensiones" (p. 7). Calcularon las correlaciones entre las escalas y los compuestos de escala de los factores del CPI y el NEO PI (Costa \& McCrae, 1985). Muchas correlaciones (significativas) exceden el .50 . Se interpretaron los resultados como substancialmente yuxtapuestos, pero no pueden ocultar las diferencias. El CPI no contiene por ejemplo Agradabilidad.

Briggs (1992) comparó, entre otros, los inventarios de personalidad de Hogan y el NEO PI. Hogan (1986) se dirigió principalmente a la predicción de criterios ocupacionales con la ayuda de grupos de ítemes homogéneos. Distinguió 43 grupos y extrajo seis factores (las cinco grandes dimensiones se añaden entre paréntesis): Ambición (-, ninguna de las cinco dimensiones), Sociabilidad (Extroversión), Simpatía (Agradabilidad), Prudencia (Escrupulosidad), Ajuste (Neuroticismo, Estabilidad Emocional) e Intelectancia (Apertura a la experiencia). El NEO PI es (deliberadamente) más amplio que los inventarios de personalidad de Hogan. Los últimos son más adecuados para la predicción en ambientes ocupacionales.

Jackson, Paunonen, Graboni \& Goflin (1996) estudiaron el Formulario de investigación de personalidad de Jackson (1984) y las cinco grandes dimensiones. Compararon un modelo con 5 y 6 factores. El último "Escrupulosidad" fue dividido en un factor de Logro y en otro acerca de lo metódico, encontrando soporte para el segundo modelo. Este resultado, de acuerdo a los autores, tiene soporte para alentar a los investigadores a encontrar alternativas para las cinco grandes dimensiones. Dieron por 
hecho que otras investigaciones producirían más diferencias en las escalas de personalidad con las cinco grandes dimensiones.

Church (1994) comparó el Cuestionario de Multipersonalidad (MPQ) de Tellegen (1982) con el NEO PI. Las correlaciones y el factor conjunto de análisis mostró un yuxtaposisición. Catorce de las 20 escalas de MPQ correlacionaron en más de .40 con las dimensiones y/o facetas apropiadas del NEO PI. Los valores de las escalas con validez convergente más fuerte incluyen Bienestar con Emociones positivas (.54), la Potencia Social con la Asertividad (.67), el Logro y el Contral con la Escrupulosidad (.48 y .50). La Cercanía Social con el afecto (.53) y lo Gregario(.56), el Tradicionalismo con la Apertura a los valores (-.56), y la Absorción con la Estética (.57). La solución de los cinco factores si correspondía a las cinco dimensiones y explica el $48.3 \%$ de la varianza. El primer factor es el Neuroticismo (MPQ: Reacción al Estrés y el NEO PI: Neuroticismo) y la segunda es Extroversión (NEO PI) y el MPQ (PEM Comunal). El tercero se interpreta como Apertura a la Experiencia (NEO PI) y la Absorción (MPQ). La Agradabilidad (NEO PI) es el cuarto que corresponde con el MPQ: Potencia Social, esto es, dominante y agresivo. La Escrupulosidad es el quinto factor que corresponde con el logro MPQ. Church concluye (p. 905): "Estos resultados de los cinco factores muestran que las escalas primarias de Tellegen pueden organizarse adecuadamente bajo, o representado por el modelo de las cinco dimensiones". El autor aparentemente hace un esfuerzo para encontrar similitudes y se impresiona por las semejanzas a pesar de las diferentes estrategias de desarrollo del instrumento. El NEO PI es deductivo, de arriba hacia abajo, desde las amplias dimensiones hacia las facetas; por su parte, Tellegen utilizó un procedimiento empírico de abajo hacia arriba para formar los grupos y los factores.

Wiggins (1979) utilizando el diseño de facetas de Foa (1965) trató de delinear rasgos interpersonales. Lo circunflejo se formó por dos dimensiones: Amor y Status y contenía ocho segmentos. Para cada segmento se desarrollaban escalas de adjetivos como marcadores de los principales vectores del dominio interpersonal. De Raad (1995) demostró que las dos dimensiones (Amor y Dominancia) corresponden a las dimensiones I y II de los cinco factores: Extroversión y Agradabilidad. 
Ostendorf \& Angleitner (1993) compararon el NEO PI con el cuestionario Zuckerman-Kuhlman (ZKPQ) y PPQ el cuestionario de personalidad profesional). Encontraron un considerable traslape, sin embargo, el ZKPQ carece de Apertura y en el PPQ el Neuroticismo está ausente. El NEO PI es el instrumento más amplio. Las otras escalas contienen más significados específicos.

Uno puede afirmar que John ya predijo en su revisión de 1990 hasta un cierto punto estos resultados. Comparó las cinco grandes dimensiones tomando como base lo conceptual y no lo empírico con los otros constructos de personalidad. Categorizó las dimensiones, los componentes y los factores de 13 teóricos (Bales, Block, Buss \& Plomin, Nollet, Gough, Guilford, Hogan, Jackson, Myers-Briggs, Tellegen y Wiggins) en las cinco grandes dimensiones (p. 89). Muchos de los resultados de los estudios empíricos confirman su análisis conceptual, pero no afirman que los constructos de los teóricos pueden intercambiarse por las cinco grandes dimensiones. Argumenta que tiene sentido en la comunicación sobre el significado de los constructos para comparar los mismos con los cinco basados psicoléxicamente. Los estudios anteriores están sesgados por encontrarse similitud. Excepto por el comentario, basado conceptualmente, de John sobre las cinco dimensiones entre muchas otras escalas, ningún soporte empírico muestra que las cinco grandes dimensiones ganen un rol central, por ejemplo hay correlaciones más altas con muchas otras escalas de personalidad, pero cuando éstas son comparadas mutuamente entre sí proporcionan correlaciones más bajas.

Finalmente, si las cinco dimensiones son fuertes, entonces también serán comparables transculturalmente. La validez de las dimensiones ha sido demostrada en las muestras representativas de adjetivos descriptivos de personalidad en Inglés americano (Goldberg, 1990), en alemán (Ostendorf, 1990) y en holandés (Brokken, 1978 y De Raad, 1992). Para añadir dos recientes ejemplos: Shemelyov \& Pokhil'ko (1993) reportaron un estudio psicoléxico y construyeron grupos de 2090 términos rusos de rasgos. Utilizando las 75 categorías de Goldberg, concluyeron que existe una similitud entre las cinco grandes dimensiones y los seis factores rusos más importantes. Sin embargo, la secuencia no es la misma. Las dimensiones rusas, en la secuencia de la cantidad de la varianza explicada son: 
Agradabilidad, Intelecto, Surgencia (Extroversión), Escrupulosidad, Estabilidad Emocional y la sexta dimensión tiene cargas de -.60 en Agradabilidad .46 en Surgencia, .41 en Estabilidad Emocional y -.32 en Intelecto. Los autores consideran sus hallazgos más que concordantes con Goldberg. No obstante, al terminar su artículo comentan una diferencia en el contenido (p.15): “... la estructura del léxico ruso (y la correspondiente estructura de las percepciones sociales) es más dependiente de los significados afectivos que en el Inglés americano". Añaden: "Si tomamos en cuenta las condiciones estresantes de la vida social rusa hoy en día, esto es lo único a esperarse".

Narayanan, Menon \& Levine (1995) condujeron un estudio en dos universidades hindúes, en dos muestras de estudiantes. De las descripciones libres, se tomaron los 40 adjetivos más frecuentemente mencionados, de los diez primeros adjetivos uno o más podían categorizarse en las cinco grandes dimensiones. El análisis de los componentes principales en los 40 adjetivos produjeron las cinco dimensiones. No obstante, hubo algunas diferencias en el tamańo de los factores y en la clase de descriptores que podían categorizarse en cada una de las dimensiones. La Extroversión y la Agradabilidad son los primeros en los Estados Unidos de América y Europa, mientras en la India el factor más grande es la Escrupulosidad. Una técnica de incidencia crítica en la cual los ítemes que típicamente pertenecen a las cinco dimensiones se elicitan, ofrece confirmación de la correspondencia entre las dimensiones de personalidad angloamericana y la hindú. La Escrupulosidad y la Agradabilidad son observadas más a menudo en los incidentes críticos hindúes que la Extroversión y la Estabilidad Emocional. Los investigadores transculturales se impresionaron por la coincidencia y al mismo tiempo que reportan las diferencias.

Esta sección muestra que las cinco grandes dimensiones se interpretan como fuertes, tanto por el método de la varianza como por mantener su significado en los estudios correlacionales con otras escalas generales de personalidad. Existe algún soporte empírico para tal afirmación. Por otro lado, las diferencias por el método (por ejemplo, Presley et al., 1994: juicios de los padres y maestros), y entre los constructos (por ejemplo, la recomendación de Jackson et al., 1996)y entre las culturas (Narayanan et al, 1995) son subexpuestos. Este deseo de encontrar la semejanza impidió el 
enriquecimiento del significado del constructo al analizar completamente las diferencias. No obstante, el significado de constructo de las cinco dimensiones está enriquecido por las facetas añadidas. Como quiera, estas son consecuencia del análisis de los adjetivos y no de la comparación con otros significados de constructo.

\section{Validez predictiva de las cinco grandes dimensiones}

Además de comparar mutuamente los constructos de la personalidad para obtener significado, tradicionalmente los constructos derivan el mismo en una considerable cantidad, de su poder de predecir conductas pertinentes. ¿El perfil de las personas en las cinco grandes dimensiones permite predecir conductas relacionadas, tales como estatus de identidad, relaciones sociales, desempeño escolar y laboral, elección vocacional, trastornos conductuales y de la salud? Recientes ejemplos representativos de esta investigación serán descritos a continuación.

Clancy \& Dollinger (1993) predijeron los estatus de identidad de 198 estudiantes de pre-grado tomando como base sus puntajes en las cinco grandes dimensiones del NEO PI. Teniendo como base los hallazgos empíricos previos, esperaban una relación negativa entre los estatus de identidad de Exclusión y Apertura. Se predijo que el logro de identidad correlaciona, aunque baja, con Neuroticismo y alta con Escrupulosidad; La Moratoria y la Difusión deberían relacionarse con el Neuroticismo. Las correlaciones fueron significativas, pero modestas. El Neuroticismo correlacionaba con la Moratoria, la Difusión y el Logro de Identidad . 35, .25 y -.27 respectivamente. La Extroversión y el logro de identidad correlacionaron significativamente (.35) y E correlacionó $-.30,-.19$ con Difusión y Moratoria. La Apertura y la Exclusión fueron correlacionadas -.50. La Escrupulosidad fue negativamente relacionada a la Moratoria y la Difusión (-.22 y -.38) y positivamente con el Logro de Identidad (.30). Este estudio es correlacional, así que la dirección entre las cinco grandes dimensiones y los estatus de identidad no es claro. ¿Los rasgos existentes moldean el curso de la identidad o causan cambios en los estatus de identidad en el perfil de la personalidad? Además, los constructos son de diferentes niveles (McAdams, 1994b) y consecuentemente no fáciles de conectar: un constructo de diferencia individual no es constructo del 
desarrollo. Clancy-Dollinger (1995) utilizaron otro modelo para la evaluación del status de la identidad: los estilos de identidad. La información orientada y el estilo de identidad normativo fue positivamente relacionadc a la Extroversión, Agradabilidad y a la Escrupulosidad. La Apertura y e! estilo orientado de información se correlacionaron. El Neuroticismo se correlacionó con los estilos difuso/evitativo. Las correlaciones fueron más modestas. La Apertura y el estilo orientado de Información correlacionaron .40. Los otros valores se encontraban entre .15 y .36 .

¿Puede uno predecir desde el perfil de personalidad en las cinco grandes dimensiones cómo se relacionaran íntimamente las personas cuando adulros? Shaver \& Brenan (1992) predijeron el estilo de Apego de los adultos a partir de sus perfiles de personalidad en el NEO PI. Los análisis de regresión múltiple mostraron que la Seguridad fue predecida mejor por un bajo Neuroticismo y alta Extroversión (las facetas bajo nivel de ansiedad y alto nivel de afecto). La Evitación se predijo por (baja) Agradabilidad, (alto) Neuroticismo (especialmente depresión), y (sentimientos de) baja Apertura. El estilo Ansioso/ambivalencia fue precedido por (alto) Neuroticismo (depresión) y (bajos valores de) Apertura. Las correlaciones fueron significativas pero modestas, variando desde .14 hasta .39 (ver $\mathrm{p}$. 540) Existe una relación entre Apego y las dimensiones de la personalidad (y facetas), pero no pueden reemplazarse mutuamente.

John, Caspi, Robins, Moffit \& Stouthamer-Loeber (1994) calcularon correlaciones entre las cinco grandes dimensiones (construyeron del CCQ: "CCQ versión de las cinco grandes dimensiones" de 48 items) y el desempeño escolar (lectura, escritura, ortografía y matemáticas fueron calificados por sus profesores en una escala de cinco puntos) de niños de 1213 años de edad. La dimensión Escrupulosidad correlacionó significativamente con los cuatro logros escolares (.20 a .24). La Apertura también correlacionó significativamente con el desempeño escolar (.17 a .22). No se encontraton relaciones con Extroversión, Agradabilidad y Neuroticismo. Las correlaciones son modestas; sin embargo, dos dimensiones de personalidad si juegan un rol importante de juicio de los maestros sobre los logros escolares de los muchachos.

Gottfredsen, Jones \& Holland (1993) conectaron las cinco dimensiones de personalidad a las seis dimensiones de Interés Vocacional de Holland. 
Reclutas mujeres y hombres de un centro de entrenamiento de la marina de los E.E.U.U. ( $n=479$ y 246 respectivamente) sirvieron como sujetos. Las correlaciones entre las escalas del NEO PI (y facetas) y los Intereses Vocacionales (Realistas, Investigativo, Artístico, Social, Empresarial, Convencional) fueron calculadas. La Extroversión se relacionó a los intereses Social y Empresarial; la Apertura los intereses Investigativo y Artístico y el Control (Escrupulosidad) a intereses convencionales. El Neuroticismo tuvo correlaciones negativas bajas para todos los intereses. Las correlaciones son "modestas" y van desde .03 a .53 como lo admiten francamente los autores (ver p. 520-523).

Un completo meta/análisis del poder predictivo de las cinco dimensiones de personalidad para el desempeño laboral fue llevado a cabo por Barrick \& Mount (1991). Distinguieron tres criterios de desempeño (habilidad laboral, habilidad de entrenamiento y datos personales) en cinco grupos (profesionales, políticos, gerentes, vendedores y trabajadores calificados y semi-calificados). Los autores son conscientes del hecho, a menudo demostrado empíricamente, que la personalidad es un predictor muy modesto del desempeño laboral. Ellos consideran a la taxonomía de las cinco grandes dimensiones como un esquema útil, puesto que organiza muchos rasgos de personalidad. Afirman que el consenso en número y contenido de los factores no son completos. No obstante, las cinco dimensiones y otras escalas son relativamente independientes de las capacidades cognitivas; por consiguiente pueden contribuir a la predicción del desempeńo laboral independientemente de las capacidades cognitivas. Guiados por los datos empíricos y la especulación, esperában una correlación de la Escrupulosidad y la Estabilidad Emocional con el desempeño laboral. Además, esperaban interacciones de la Dimensión " $x$ " (con el desempeño " $x$ "; con relaciones empleado-grupo, como por ejemplo, $E$ interactúa con el grupo empleado (gerencia y ventas) y $\mathrm{O}$ interactúa con el tipo de desempeño laboral (habilidad de entrenamiento).

Los autores utilizaron 117 estudios de validez realizados entre 1952 y 1988 con muestras que varían entre 13 y 1401 participantes. Las escalas de personalidad fueron clasificadas en las cinco dimensiones utilizando la descripción de Digman (1990) de las cinco dimensiones, seis jueces codificaron las escalas de acuerdo a su descripción. Una cantidad de 191 
escalas fue dividida entre las cinco dimensiones: $39 \mathrm{~N}, 32 \mathrm{E}, 31 \mathrm{O}, 29$ A, 32 C y 28 misceláneas ${ }^{3}$. De acuerdo a algunas recomendaciones de Schmidt \& Hunter (1977) (el concepto de generalización de validez) se corrigió la varianza de los coeficientes de validez para el error de muestreo, la restricción del rango y se atenúo para el error de medición. Los diferentes tipos de validez de los predictores -si estaban disponibles-fueron tomados de los manuales, sino fueron estimados. El resultado para la dimensión Escrupulosidad fue significativa (un $\mathrm{r}$ estimada de .20 a .23 ); para Neuroticismo la correlación fue estimada (-.13 a .12). Estas correlaciones son de orden cero y no correlaciones múltiples, ni canónicas. La Agradabilidad no predijo el desempeño laboral. La magnitud de las correlaciones estimadas de los puntajes reales fue pequeña $(r<.10)$. El hallazgo notable fue la validez predictiva de Escrupulosidad para todas las medidas de los grupos de empleados y de desempeño laboral. La Extroversión fue válida para los tipos de criterio, pero sólo para los gerentes y vendedores. La Apertura predijo la habilidad de la capacitación, pero no de la destreza laboral y en los datos personales. Los autores se sorprenden por la falta del poder predictivo del Neuroticismo y sugieren la posibilidad de restricción del rango; es decir, los empleados son seleccionados por bajos puntajes de Neuroticismo (rango estrecho). La Agradabilidad no es un predictor pertinente, ya que el hecho de ser juzgado como cortés, confiable, honrado, bondadoso no predice el desempeńo laboral. Un estudio holandés de Van Dam (1996) apoya este hallazgo. Su estudio muestra que las personas que seleccionan personal utilizan las cinco dimensiones de personalidad. En sus decisiones, no obstante, la Agradabilidad no juega ningún rol, mientras la Escrupulosidad si.

¿Existe una relación entre las cinco dimensiones y la salud (percibida)? Marshall, Wortman, Vickers, Kusulas \& Hervig (1994) están de acuerdo con la presunción que las características de personalidad pueden influir en la vulnerabilidad para las enfermedades. En la literatura sobre personalidad, se consideran diversos constructos para la salud; por ejemplo, el optimismo, el autodominio, la autoeficacia generalizada, la expectativa por el éxito, la fe en sí mismo y "la fortaleza". Utilizando una muestra de 296 reclutas

3 Siglas de las dimensiones en Inglés. 
de la marina, los autores relacionaron el NEO PI con 16 instrumentos de salud pertinentes. Estos últimos fueron primero enviados a un análisis factorial principal, utilizándolas correlaciones múltiples cuadradas como estimados comunales y la rotación varimax. Tres factores explicaban el $61 \%$ de la varianza y fueron nombrados: I Optimismo, esperanza, control interno, autoestima y fe en las habilidades de uno; II Expresión Racional versus Expresión Emocional y III Inhibición y Meditación. Estos se relacionan a las cinco dimensiones: el Factor I se correlacionó con N, E y $\mathrm{C}$; el II con $\mathrm{N}$ y A y el III con $\mathrm{N}$ y O. Las correlaciones varían de .15 a .64; el Neuroticismo tenía en particular varianza en común con el control Optimista.

Además, Costa \& McCrae (1991) argumentaron que los patrones de puntaje en las cinco grandes dimensiones pueden tener sentido al analizar las escalas del trastorno de personalidad. Los problemas relacionados a la personalidad en las áreas interpersonal, experiencial, actitudinal y motivacional pueden describirse y entenderse mejor con la ayuda de los patrones de puntajes en las cinco grandes dimensiones. Para dar un ejemplo, el patrón de un puntaje $\mathrm{E}$ alto $\mathrm{y}$ un $\mathrm{A}$ bajo pueden referirse a un trastorno de personalidad narcisista. Estas hipótesis tienen aún que someterse a prueba empírica.

John et al. (1994) exploró la relación de las cinco dimensiones de personalidad (Formato cinco grandes dimensiones CCQ) con la delincuencia en niños de 12 a 13 años de edad. Se distinguieron cuatro niveles de delincuencia (de vandalismo y robo en casa a ingreso forzado, armamento pesado y venta de drogas). Un análisis de varianza de una vía mostró efectos de Agradabilidad y Escrupulosidad. Un alto nivel de delincuencia estaba acompañado de niveles inferiores de A y $\mathrm{C}$. Los efectos más débiles aparecieron en Extroversión y Apertura (faceta de búsqueda de sensación): un alto nivel de delincuencia en E y O. No hubo efecto de Neuroticismo. Los efectos son significativos pero la varianza explicada se estima como modesta (si se calcularan los cuadrados Omega en lugar de los valores F).

Adicionalmente, Heaven (1996) asoció las cinco dimensiones con la violencia interpersonal y vandalismo/robo autorreportado en 216 estudiantes de secundaria. En este estudio, la dimensión A y en un grado menor 
las dimensiones $\mathrm{C}$ y $\mathrm{N}$ (en contraste con el estudio de John et al. de 1994) fueron relacionadas a violencia y vandalismo. Las correlaciones fueron modestas, variando de .19 a .46 .

Finalmente, las dimensiones de personalidad se relacionaban con patología infantil. John et al. (1994) distinguió dos conductas problema: internos (ansiedad, quejas somáticas, retraimiento social) y externos (agresión, robo, desatención, conducta impulsiva e hiperactiva). Estos últimas eran más Extrovertidos, pero no Agradables, ni Escrupulosos. Los primeros eran Neuróticos y no Escrupulosos. Los autores no calcularon las correlaciones sino las diferencias. Sin embargo, la varianza explicada por las dimensiones de personalidad se estima como modesta. En un estudio holandés, Goedhart \& Treffers (1992) analizaron el perfil de personalidad de los niños deprimidos. Estos niños se diferenciaban de los niños normales del grupo de control. Los primeros puntuaron bajo en A, E y C. No se encontraron diferencias en $\mathrm{O}$ y $\mathrm{N}$. Esto último sorprendió a los autores.

Coolidge, Becker, DiRito, Durham, Kinlaw \& Philbrick (1994) determinaron la relación entre las cinco grandes dimensiones y los trastornos que son enumerados en el Eje I del DSM-III-R en 233 estudiantes. Los trastornos fueron Antisocial, Evitativo, Limítrofe, Dependiente, Histriónico, Narcisista, Obsesivo, Compulsivo, Paranoide, Pasivo agresivo, Esquizoide y Esquizotípico. Se encontraron modestas correlaciones significativas y canónicas. Entre las cinco grandes dimensiones y los trastornos fueron: N .48, E .31, C .22 y 0.12 .

Esta sección muestra que las cinco grandes dimensiones se usan frecuentemente para predecir conductas pertinentes en niños y adultos. Se informan muchas relaciones significativas. La varianza explicada es modesta. Por consiguiente, la pertinencia para la predicción individual es limitada. Las cinco grandes dimensiones no hacen un trabajo especial, comparando con las escalas ya existentes. Estos estudios son de procedimiento similar a los muchos estudios correlacionales de CI en muchas otras conductas. Las correlaciones de las cinco grandes dimensiones con las conductas son, no obstante, considerablemente inferiores. Se carece de una explicación para las primeras correlaciones. 


\section{Estatus teórico de las cinco grandes dimensiones}

¿Cómo se evalúa el estatus teórico de las cinco grandes dimensiones en psicología? Las cinco grandes dimensiones no se originan en una teoría, ni en un enfoque tipo facetas en la persona (ver por ejemplo, Foa, 1965) y no son por consiguiente constructos explicativos. Pueden distinguirse tres perspectivas con respecto a las cinco grandes dimensiones: la léxica, la semántica y la social.

La perspectiva léxica utiliza el diccionario, se estima que contiene el $10 \%$ del léxico. Este se considera desde un punto de vista realista; es decir, las expresiones del lenguaje sobre las diferencias entre los individuos se consideran como la base de las diferencias reales en las características de las personas. Para el estudio empírico tienen que hacerse las selecciones del diccionario y los criterios describirse de la manera menos ambigua posible, pero no existen reglas convincentes y consecuentemente pueden formarse diferentes bases de datos (De Raad, 1996, en prensa). Los criterios usados definen a la persona en el sentido léxico y existe consenso que éste debe considerarse como la fuente para las diferencias individuales en rasgos, lo que permite la operacionalización de un aspecto de las diferencias de personalidad. La verdad después de todo, le pertenece a la realidad y no a una operacionalización específica como destaca Hofstee (1994). Esta perspectiva ha ganado un lugar en la psicología, especialmente porque produce una taxonomía general útil para describir las diferencias individuales.

De acuerdo a la perspectiva semántica, no obstante, las dimensiones se refieren a las características lingüísticas. Si uno estudia la similitud semántica del lenguaje de la personalidad, por ejemplo los adjetivos, uno llegará a la estructura de las cinco dimensiones (Mulaik, 1964). La estructura es un sujeto de análisis semántico de significados y no de un estudio psicológico. En las décadas del sesenta y del setenta, esta perspectiva retardó la investigación sobre las cinco dimensiones de la personalidad. Puede añadirse que el léxico es acerca del lenguaje cotidiano. La ciencia, se dice, necesita un lenguaje técnico claro y no el caprichoso que la persona común habla todos los días. Se considera peligroso construir el conocimiento con la ayuda de tal base defectuosa. Recientemente, no obstante, Hofstee (1994) argumentó justo lo contrario. En su provocante artículo, él defiende 
que la definición de la persona debe ser dada por gente común. Sus respuestas estarán mediadas por el analista psicométrico, por ejemplo, el analista factorial. Si bien la gente común no es experta, su gran número superan a los teóricos.

Una pregunta interesante para la perspectiva de Hofstee es ¿cómo se desarrollará en el futuro la psicología de la personalidad como ciencia? ¿Cambiará sólo como consecuencia de diferentes resultados del siguiente referéndum en una muestra representativa? ¿De ahora en adelante la investigación en personalidad es una encuesta de opinión? ¿Existe un lugar para el psicólogo/teórico de la personalidad? La discusión sobre el rol del lenguaje común en psicología parece haber ingresado a una nueva etapa. Las hipótesis del significado léxico realista y del significado semántico tienen que confrontarse. Semin (1990) y Semin \& Krahe (1992) argumentaron, sin embargo, que no tiene sentido exagerar la dicotomía entre la taxonomía de los rasgos como una característica interna de la mente y como una peculiaridad del mudo real externo de las personas, porque la gente siempre está conectada al mundo social y cultural. El lenguaje juega un rol importante en esta conexión.

La perspectiva social es sobre el valor del juicio social humano, y los rasgos de las personas. Se afirma a menudo que el juicio social, en general, y la atribución de rasgos, específicamente por medio de los reportes de uno mismo y de los demás, son aturdidos por las preocupaciones de los jueces. Se dice que sus juicios sólo están ligeramente relacionados con las características reales de las personas juzgadas. Desde la década de los cincuenta hasta mediados de los ochenta, se analizó críticamente el proceso del juicio social. Las revisiones de Cronbach (1955) y Bruner \& Tagiuri (1954) indicaron los prejuicios y la falta de precisión del juicio humano. Como consecuencia, se desarrollaron muchos modelos normativos de decisión, que muestran (Van Dam 1996) que el juicio humano era un pobre estadístico intuitivo (Nisbett \& Ross, 1980) y un procesador de información limitado y falible (Hogarth, 1980; 2da edición 1987). Fue en ese período que no se usaron los reportes sobre uno mismo y de los demás para encontrar la estructura de los rasgos de personalidad. Además, Mischel (1968) argumentó que los rasgos predecían mal los criterios pertinentes; las situaciones explicaban más la varianza en conductas. Esta perspectiva 
sobre los rasgos combinada coombinada con la crítica del proceso del juicio social no fue fructífera para las cinco grandes dimensiones. Los estudios sobre las teorías de personalidad implícitas y estereotipos fueron conducidos para investigar como la maquinaria mental humana conectaba los rasgos de las personas. No hubo pretensión para descubrir las dimensiones básicas que podían construirse a partir de estas teorías implícitas. El objetivo fue más bien demostrar la frágil conexión del juicio humano y de la realidad. Estos ataques de los rasgos provocó reacciones que condujeron a su rehabilitación (Funder, 1987, 1993). El observó: "Alguien dijo una vez que lo que hace a un oso bailarín tan impresionante, no es el hecho de que baile bien sino de que baile. Estoy impresionado por el juicio humano de la personalidad por casi la misma razón, no porque los juicios sean perfectos, sino porque frente a enormes dificultades, parece digno de destacar el que se las arreglen para tener algo de precisión." (1989, p. 212).

Esta sección muestra un debate animado donde las preguntas no son respondidas, ni resueltas. Se necesita una discusión adicional en la cual el status de las cinco dimensiones se compare con otros constructos (explicativos). ¿Cuál es la fortaleza del enfoque de las cinco dimensiones?, ¿cuál es su debilidad? ¿dónde está el valor adicional del enfoque léxico?

\section{Críticas históricas y recientes de las cinco dimensiones}

Tanto en su historia como recientemente, se han hecho muchas críticas a las cinco dimensiones. Iniciando con críticas recientes, uno pude referirse al artículo de Block (1995). Admite que las cinco dimensiones son un sistema descriptivo útil, pero expresa también sus dudas: ¿Es la base empírica nominal fructífera? ¿son las cinco dimensiones los constructos nucleares de la personalidad? ¿no está el enfoque demasiado orientado hacia lo variable, que carece de la motivación y desarrollo de la persona? Otro crítico reciente es McAdams (1992, 1994b), quien independientemente de Block, enumera una cantidad de limitaciones: Las cinco grandes dimensiones no son los constructos esenciales de la personalidad, no son predictores eficientes de conductas pertinentes, no contienen explicaciones, la naturaleza condicional y contextual de la conducta está descuidada (suena irónico para McAdams que las cinco grandes dimensiones se describen como "transcontextuales" y "transituacionales") y no es un programa de 
investigación atractivo. Las cinco consideran a la personalidad desde la perspectiva de un observador independiente: "... en el que el objetivo de la puntuación se vuelve un objeto de comparación en una serie de dimensiones lineales e imprecisamente condicionada " (McAdams, 1994b, p. 303). Además, las cinco dimensiones tienen respuestas para cinco tipos de preguntas sobre un extraño que están por conocer (Goldberg, 1981, p. 161).

Fuera de estas críticas recientes, en la historia de las cinco dimensiones diversos investigadores expresaron sus dudas. Estas se referían primero, a las dimensiones apropiadas: número, validez de contenido, de estructura, de constructo y predictiva. Segundo, las preguntas fueron hechas sobre el poder explicativo, la generalidad, la especificidad y la conexión con el lenguaje cotidiano y común. Tercero, se pregunto ¿qué clase de persona se describía?, ¿quién es él/ella?, ¿dónde está: en nuestras cabezas, en el exterior?; ¿es estable o capaz de cambiar?, ¿se desarrolla?

A continuación, se elaboran tanto las críticas como las sugerencias en defensa de las cinco dimensiones. Asimismo, algunas especulaciones sobre el curso futuro de la investigación de la personalidad será esbozada.

Se critica el número de cinco, lo que es parcialmente provocado por una afirmación de McCrae \& John (1992, p. 194): "Creemos que ello (la existencia de los cinco factores) es un hecho empírico, como el hecho de que son siete los continentes sobre la tierra u ocho los presidentes norteamericanos oriundos de Virginia." Como se señala más arriba, Hogan (1986) clama por 6 factores e incluye Sociabilidad y Ambición. Block (1995) señala como arbitrario el número de factores o componentes principales. Estos varían de 3 a 7 , no porque en realidad existan, pero como consecuencia de la facilidad para mantener esta cantidad de dimensiones en nuestra mente (ver por ejemplo, Presley \& Martin, 1994). Además, Block argumenta en contra del análisis factorial como técnica de reducción: si uno tiene éxito en una reducción substancial, entonces los datos son redundantes, y la ciencia se orienta sobre la información y no sobre la redundancia. Parece haber una pelea por "el espacio de personalidad".

En defensa del número de cinco, y como afirmación especulativa sobre su futuro, uno puede referirse a la discusión sobre la cantidad de factores en la investigación sobre inteligencia. La lucha por el espacio de la inte- 
ligencia está terminada, no se necesita más "una barrera de espacio". A partir de los diferentes niveles jerárquicos, pueden distinguirse un número variable de factores; no hay un sólo y único número. Se admite que el análisis factorial tiene sus limitaciones y que cinco no es un número sagrado (De Raad, 1996, en prensa). Esto no niega el hecho que al nivel de la generalidad de las cinco dimensiones, 4 a 7 factores se encuentran consistentemente en estudios de adjetivos de personalidad. Además, seis facetas específicas se añaden a cada dimensión. Hofstee \& De Raad (1993) argumentan por ejemplo en contra de Eysenck, quien defiende tres factores (algunas veces llamados los tres más grandes: Psicoticismo, Extroversión y Neuroticismo), que enfatiza demasiado el nivel jerárquico más alto. La cantidad de factores ya no es más una cuestión para el debate principal.

Se discure el contenido de los factores. Existen diferentes nombres para las cinco dimensiones y el contenido del quinto factor no es claro. Algunos autores incorporan también inteligencia, mientras otros por el contrario, están satisfechos con un perfil de personalidad independiente de inteligencia. Waller \& Ben-Porath (1987) se refieren al dudoso procedimiento de selección de escala bipolar y sugirieron que las cinco dimensiones no son del todo globales. Muchos conceptos interesantes, por ejemplo el catálogo de Gough, conceptos populares y otros constructos de personalidad no integrados. Loevinger critica el contenido de la dimensión Conciencia, porque el factor no es sensible a la diferencia entre Conformidad y Escrupulosidad. Ella se refiere al modelo de desarrollo del Ego en el cual esta distinción se hace explícita. El consenso sobre el contenido de las cinco dimensiones no se garantiza y Loevinger $(1993,1994)$ indica la incompatibilidad de su modelo evolutivo y las cinco dimensiones.

En defensa de las cinco dimensiones demasiado "molares", puede decirse que a un nivel más específico son posibles distinciones adicionales. Esas no pretenden cubrir todas las clases de características de la personalidad en cada nivel. Existe una limitación para las diferencias individuales generales, transituacionales, disponibles en la forma de adjetivos extraídos de un diccionario. La inteligencia, el procesamiento de información, el temperamento, los estilos de apego, la dependencia de campo, las actitudes, la moralidad, etc. (ver una lista de cerca de 400 componentes de personalidad de Mayer, 1995) no son explícitos y no forman parte de la base 
de datos de las cinco dimensiones. Aunque Cattell (1995) continua defendiendo los 16PF y sólo está dispuesto a admitir que existen 8 factores de segundo orden, su análisis no encaja con las prácticas analíticas factoriales contemporáneas (De Raad, 1996, en prensa).

La lucha sobre el contenido es una búsqueda por el significado, la misma lucha que tiene que darse y debe continuar. La contribución de Loevinger sobre la comparación de diferentes clases de constructos (diferencias individuales versus constructos estructurales del desarrollo). La otra comparación puede contribuir al descubrimiento del significado de los constructos para describir mejor a las personas y también contribuye también a predecir las conductas pertinentes. Probablemente, la crítica no es lo suficientemente firme, porque algunos autores enfatizan de un modo insuficiente las limitaciones del nivel de las cinco dimensiones y que estas corresponden con las otras escalas de personalidad.

En el futuro se deberá elaborar más el contenido. Comparaciones más estrictas con otras escalas de personalidad forzarán definiciones más precisas de los cinco factores. Más estudios funcionales tienen que ser llevados a cabo, en los que se estudien las interacciones de las cinco dimensiones, sus facetas y las situaciones específicas. El ser transituacional o transcontextual no es una meta final en la investigación de la personalidad. El sistema de las cinco dimensiones debe ser también capaz de adaptarse a las situaciones. Esta investigación puede aun añadirse a las cinco dimensiones, por ejemplo, en situaciones sociales diferentes, algunos factores específicos son más pertinentes que otros. Como un ejemplo de lo último está el uso de dimensiones diferentes por parte de los padres y maestros al juzgar el temperamento de los niños. Los maestros utilizaron diferentes dimensiones que los padres y fueron diferencialmente precisos al describir la conducta de los niños (Presley, 1994). En un estudio de Ter Laak (1994) los maestros usaron cuatro componentes principales para describir a sus alumnos. Fueron exactos (según se controló por observaciones de las conductas de clase de los niños) sobre las dimensiones "Conducta perturbadora" y "Persistencia en la tarea", pero no sobre "Sociabilidad". Así, en su calidad de maestros, nuestros sujetos tenían su propia estructura de dimensiones pertinentes. Esta estructura no difería individualmente entre los maestros, como una comparación de las estructuras individuales mostradas. Además, 
fueron (individualmente similares) diferentemente precisos en las dimensiones. Esta precisión diferencial con respecto a las dimensiones fue fácil y funcionalmente interpretada. Los maestros en su calidad de docentes, tienen que manejar la clase (son sensibles con los niños intranquilos) y les gusta enseñar (distinguen entre los alumnos que hacen o no hacen bien la tarea). Todos los profesores tenían esta estructura de diferencias entre los alumnos en "su mente" y esto puede compararse con las cinco dimensiones. ¿Esta estructura general se aplica parcialmente a esta situación específica de aula?

Para explicar las cinco dimensiones, se continuarán los estudios genético conductual y se ha avanzado ya mucho al respecto (Loehlin, 1992). Se encontraron los componentes genéticos substanciales, aun para la Escrupulosidad. Se espera que este factor dependa relativamente más del medio ambiente. Bergeman, Chipuer, Plomin, Pedersen, McClearn, Nesselroade, Costa \& McCrae (1993) encontraron que la Escrupulosidad tenía un componente genético sustancial. En el futuro, se enfatizará (y tiene que ser) que los componentes sustanciales de las cinco dimensiones dejen suficiente espacio para las influencias ambientales (Ravelle, 1995).

Debido a que las cinco dimensiones están establecidas sólidamente, en el futuro las interacciones de éstas y las facetas serán utilizadas en estudios de personas en situaciones específicas. Una limitación de los estudios, presumiblemente es que capitalizan la estabilidad y descuidan el cambio.

Las cinco dimensiones descansan sobre el análisis de componentes principales, del factorial y de la rotación varimax. Las técnicas son usadas mayormente en un modo exploratorio y buscan la "estructura simple". Loevinger (1994) critica este modelo de "columnas y filas" para simplificar la realidad humana. Los resultados de los análisis en realidad muestran que los adjetivos de personalidad a menudo cargan sustancialmente en dos factores. Como una defensa en contra de esta simplificación, uno puede señalar el hecho de que justo por este fenómeno, Hofstee y De Raad (1993) propusieron un modelo circunflejo-factorial integrado. Recientemente, se han usado análisis factoriales confirmatorios (Jackson, Paunonen, Fraboni \& Goflin, 1996); no obstante, sus resultados se centraron en los análisis exploratorios. Puede añadirse, como una defensa, que cada modelo paga 158 
un precio a la realidad por deformarla, pero al mismo tiempo gana una comprensión parcial de la misma. El comentario de Loevinger (1994) se refiere también al hecho de que las prácticas analíticas factoriales en la investigación no cubren el desarrollo de "estructuras" esto es: los modos de pensar, sentir y comportarse. Si los investigadores no pretenden encontrar "estructuras", entonces su reproche es injustificado; de lo contrario, Loevinger cuenta con un argumento. Se necesita un juicio equilibrado sobre las imposibilidades de las cinco dimensiones para estudiar el cambio y desarrollo de la personalidad. Los altos coeficientes de estabilidad según Costa \& McCrae (1994) sugieren erróneamente que el cambio en la investigación es superfluo. Es también necesario cambiar los conceptos para estudiar a la personalidad.

En el futuro, otros modelos multivariados serán usados para estudiar la estructura. Es probable que los resultados del modelo analítico factorial reaparecerán con otras técnicas. El estudio del cambio de personalidad necesitará otros constructos y modelos; las cinco dimensiones son apropiadas para estudiar la estabilidad de la estructura y del contenido.

Las cinco dimensiones serán inevitablemente consideradas, tanto por los teóricos como por los pragmáticos, como instrumentos/pruebas que evalúan las diferencias entre personas sobre constructos descriptivos y para propósitos predictivos prácticos. Con respecto a la validez de constructo, la mayoría de los investigadores encontraron similitudes y diferencias con otros constructos. Generalmente enfatizaron la semejanza de diversas escalas de personalidad con las cinco dimensiones y se unen al creciente consenso (es decir, la validez convergente) de las cinco dimensiones. Habría valido la pena, si hubieran enfatizado también las diferencias (es decir, la validez divergente), porque esta última contribuye tanto a la validez de constructo como la anterior. En la investigación de la validez de constructo, la meta no es comprobar tener la razón con respecto a una relación específica, sino contribuir a los ricos matices del significado de los constructos psicológicos (ver Messick, 1989; Hofstee, 1994). El cuadro de la validez de constructo que surge de los estudios se juzga como de un sólo lado. Es preferible mayor discusión sobre las diferencias sutiles y reales entre las escalas de personalidad y las cinco dimensiones. En el futuro habrá más atención para las diferencias de las cinco amplias dimensiones y otros constructos. Esto no 
será un ataque hacia las cinco dimensiones, sino una lucha por la elaboración del significado. Además, tiene que darse atención a los diferentes niveles del estudio de la persona y sus conexiones (ver McAdams, 1994; Pervin, 1994). Es infructuoso declarar los diferentes niveles como paradigmas separados o programas de investigación o hipótesis del mundo (Lakatos, 1978; Pepper, 1942). Estos conceptos infructuosos implican que no existe comunicación posible entre los niveles y los programas de investigación. Dos de los cuatros (organísmicos y mecanicistas) fueron retratados como inconmensurables en la psicología del desarrollo (Reese \& Overton, 1970): Esta bifurcación no ha promovido la psicología del desarrollo.

Con respecto a la validez predictiva, muchos estudios muestran relaciones significativas muy modestas. En cuanto a esto, los resultados de las cinco dimensiones no se desvían de los resultados de otras escalas de personalidad y las esperanzas de coeficientes de validez predictiva más altos son (y probablemente serán) en vano. En el futuro, también permanecerá como una cuestión seria para los psicólogos teóricos y pragmáticos, "por qué las diferencias individuales en personalidad contribuyen tan poco al predecir los criterios pertinentes tales como salud, desempeño escolar y laboral y trastornos conductuales? El valor adicional de las cinco dimensiones como un instrumento predictivo no se muestra aun. Posiblemente el científico social debe admitir en el futuro, que la predicción usual (con modelos lineales y hasta con otros modelos) no es el elemento más sólido de su ciencia, predicen por encima del azar para un grupo (no para una persona) y la varianza explicada es modesta. En consecuencia en los casos individuales, se cometen muchos errores. Hofstee (1994) es crítico sobre la predicción individual como la meta de la psicología de la personalidad. De acuerdo a él, este tipo de predicción (usa como ejemplo si el sol brillará cuando cumpla 60 años) no son parte de la ciencia. No obstante, la predicción individual forma parte de un trabajo mucho más práctico. Los psicólogos necesitarán la teoría y el conocimiento práctico para mejorar la predicción individual o por lo menos para ganar la comprensión sobre por qué la predicción individual es difícil: bajo qué condiciones, con qué personas y con qué constructos.

Las cinco dimensiones son una taxonomía descriptiva de los adjetivos de personalidad extraídos de un diccionario. Una taxonomía no es una 
teoría o conjunto de constructos teóricos. En defensa de las cinco dimensiones, uno puede afirmar que puede formar el punto de partida para los constructos psicológicos. Una taxonomía es más exitosa y valorada en otras ciencias; por ejemplo, en biología. Snow (1973) ha escrito acerca de la teoría, que va desde las hipótesis derivadas de la teoría axiomática hasta las hipótesis operativas y las suposiciones; todas pertenecen a la empresa científica. Aun los fuertes adherentes no pretenden que las cinco dimensiones constituyan una teoría. Así, el reproche no son teóricas es cierto, pero injustificado. El paso de la taxonomía a los constructos teóricos es un reto para el futuro.

El procedimiento extensivo requiere una lista completa de palabras pertenecientes a la personalidad. En cuanto a esto, existe una abundante crítica justificada. Los criterios para seleccionar las palabras no son claros (De Raad, en prensa, 1966) y hasta una muestra representativa del diccionario no cubre el léxico completo, puesto que nuestro lenguaje es viviente, dinámico, cambiante. Se dice repetidamente que Cattell seleccionó las escalas de un modo que no pudiesen reconstruirse en retrospectiva (John \& Angleitner, 1988). No obstante, estas escalas fueron centrales en la construcción del 16PF y el NEO PI desarrollado por Costa \& McCrae (1985). El manejo de muchas palabras (cerca de 18,000) por Allport y Odbert (1936) y Norman (1963) requieren de decisiones. Dependiendo de éstas, se formaron las diferentes bases de datos. No existen reglas generalmente aceptadas y obligatorias para las decisiones de selección. En defensa de este procedimiento defectuoso, puede decirse que el resultado era bueno, aunque los procedimientos no eran inmaculados. Los datos empíricos demuestran esto, porque al usar reglas diferentes, poco claras y hasta bases de datos diferentes, se establece un cierto consenso. Será superfluo en el futuro trabajar con una nueva lista completa, por lo menos dentro del corto plazo. El consenso es firme y nuevos estudios no cambiarán repentinamente el número y la estructura de las cinco dimensiones.

Las cinco dimensiones se deducen de una rigurosa reducción del léxico. Como consecuencia son generales. Además, existe la posibilidad de que las tres más grandes de Eysenk, sean un Gran Factor. Podría tratarse de uno evaluativo: Bueno-Malo o Verdadero-Falso. General significa no específico y por consiguiente las cinco dimensiones no corresponderán 
firmemente con las características específicas. El reproche que las dimensiones son demasiado generales es cierto, pero no justificado. Estas están destinadas a ser generales y sólo recientemente se añadieron 6 facetas a cada dimensión.

El lenguaje común se caracteriza a menudo como inapropiado para la psicología científica. Las cinco dimensiones se derivan del lenguaje común; sin embargo, el estudio psicológico del lenguaje común puede ser defendido. Su uso no implica que lo que las personas digan sobre otras personas tiene que ser considerado como cierto. Se usa sólo para construir un espacio común (el hiperespacio de las cinco dimensiones). Cada afirmación individual sobre las personas puede compararse con este espacio común, que se construye de las oraciones de un grupo representativo. Así, no puede afirmarse que $\mathrm{X}$ dice la verdad, sino sólo que su afirmación se asemeja o difiera de esta estructura común. El lenguaje común no es el único lenguaje de la psicología. También existe el lenguaje de los conceptos teóricos, de las relaciones, teorías y hasta de los modelos matemáticos. En el futuro, estos lenguajes serán (y tienen que ser) confrontados. Deben esforzarse hacia el consenso de un modo contrafáctico. Este concepto proviene de la lógica modal e indica que la discusión entre los paradigmas es posible (ver por ejemplo, ter Laak, 1995). Esto parte de la interpretación del paradigma de Reese \& Overton (1970), que toman como su punto de vista que los paradigmas son impermeables. La partida de tal perspectiva asegura una discusión actual y el enriquecimiento de las perspectivas niveladas sobre la personalidad y las personas. Esta discusión será posible porque cada paradigma es lo suficientemente fuerte para tomar parte en la discusión. Ningún paradigma dominará de antemano, por ejemplo, el neuropsicológico, el psicodinámico, el disposicional, el fenomenológico. Para estudiar los paradigmas de las personas pueden tener "Herrschaftfreier Dialog", por ejemplo, un dialogo basado en la igualdad (Habermas, 1979).

La afirmación que las cinco dimensiones no constituyen el núcleo de la personalidad es difícil de evaluar (Block, 1995; McAdams, 1994): ¿Qué significa? Con el fin de aclarar esta diferencia, se utilizan los conceptos fenotípicos y genotípicos y la estructura superficial y profunda (Distinción de Chomsky en su gramática generativa). ¿Cómo elaborar esto de manera concreta en dimensiones de la personalidad? ¿De qué manera uno encuen- 
tra las dimensiones genotípica y profunda? Esto constituye un reto para los teóricos. Block $(1982,1995)$ suministra ejemplos de los constructos de personalidad "nucleares", "dependencia del campo, nivelaciónafinamiento" y probablemente "resiliencia del ego, control del ego". Existen niveles de pensamiento en investigación en psicología aparentemente diferentes. Si los adherentes a las cinco dimensiones no pretenden tomar posesión de o dominar otros niveles, uno debe esperar que su análisis es aceprable y defendible. Pueden tomar parte en la discusión de niveles y esforzarse de manera contradictoria por el consenso. Que esto no será fácil se muestra en la respuesta de Goldberg \& Saucier (1995) a Block (1995), en que lo caracterizaban como un brillante abogado del partido opositor. Puede afirmarse que el conocimiento suministra ejemplos de constructos de personalidad "nucleares". Se puede afirmar que el conocimiento psicológico probablemente no crece según el éxito obtenido en un juicio, (como la lucha de los paradimas), sino según la lucha por el significado al analizarlo en diferentes niveles (las teorías populares y las teorías implícitas, los conceptos teóricos y teorías y los modelos matemáticos para la conducta y por comparaciones intranivel (por ejemplo, los enfoques de la matriz multimétodo-multirasgo según son explicados elegantemente por Campbell \& Fiske, 1959). En su respuesta a la crítica de Block, Costa \& McCrae (1995) ofrecen resistencia a algunos de los comentarios, pero no pueden dejar de lado algo de retórica. Al comparar a Block con Einstein, se argumenta en contra de la teoría cuántica: "Sus contribuciones (los de Block) a la teoría de la personalidad y a la evaluación son imperecederos, pero sus argumentos contra el MCF (el Modelo de los Cinco Factores) no pueden soportar el peso de la evidencia en su favor." Block (1995) a su vez, no pudo tampoco evitar la retórica. Esta disputa no contribuirá a desarrollar el conocimiento, si uno presume que la búsqueda por el significado en el sentido de la validez de constructo no se encuentra bien avanzado por la retórica de las salas de justicia.

El estatus de las cinco dimensiones es el de una taxonomía extensa bien elaborada y fácilmente accesible, provista de muchos datos empíricos. No es probablemente un paradigma o un programa de investigación en el sentido de Lakatos (1978). Si este es un juicio correcto (sólo el futuro lo sabrá), entonces las reglas del programa científico no se aplican. El convertirse en un programa implicaría, que si las cinco grandes dimensio- 
nes atraen a los investigadores (lo hace), contiene algunas creencias centrales incontestables investigadores, constructos (este es un serio problema para la investigación de las cinco grandes dimensiones, pero no es una limitación), incorpora muchos datos nuevos fácilmente y enriquece la comprensión de los datos antiguos (esto ocurre moderadamente), si hay discusión con paradigmas similares y diferentes sin la retórica de una escuela aislada, cuyos miembros son sólo comprendidos por pupilos y no por extraños. En el momento actual, es el estudio de la taxonomía de las "personas" dimensiones (como una abstracción, justo como se usa en expresiones tales como "Dem Volke gewidmet", "Para las personas"). Esta taxonomía está construida cuidadosamente y se volverá eventualmente en parte de las (muchas) escalas de personalidad para medir las diferencias individuales. Para ser especial y no ser una de las tantas requiere más estudios de validez divergentes y convergentes y una búsqueda por las limitaciones de las cinco dimensiones en relación a las características específicas.

Existe también crítica sobre la clase de persona que las cinco dimensiones describe. Tiene que destacarse de antemano que el psicólogo tiene que construir muchas "personas" y una nueva "persona" es una ganancia si contribuye al conocimiento. Este requerimiento no puede ser el que una teoría específica o descripción crítica no se dirige a "todas las personas", dado que tiene que ser construida por perspectivas divergentes. A continuación se analiza a "la persona de las cinco dimensiones".

La investigación es orientada por las variables, y no por la persona individual y su desarrollo. La estructura de las cinco dimensiones es un mínimo común múltiplo al cual todos (como grupo representativo) contribuimos de un modo democrático. El análisis factorial nos da una estructura de pesos y formas iguales, que nosotros mismos como personas individuales no necesariamente utilizamos y/o "poseemos". Todas las relaciones y las predicciones basadas en los perfiles sobre las dimensiones se computan para minimizar una suma de errores (en modelos lineales: para minimizar la suma de cuadrados) para un grupo y no para una persona individual. Esta crítica es cierta, pero no justificada., si la pretensión no es describir a la persona individual y su desarrollo. Por otro lado, tampoco se justifica descuidar a la persona como individuo cambiante/en desarrollo. 
Las cinco son las dimensiones, que a uno le gustaría conocer de un extraño, por ejemplo, un nuevo vecino: ‘hasta que punto es el/ella Extrovertida, Agradable, Escrupuloso, Neurótico y Culto? Es bueno conocer a su vecino por supuesto, pero uno no quiere saber sus motivos, planes, vida e historia. Hofstee (1994) considera esto como ventaja. "Por definición, el estudio científico de la persona es público" (p. 155). No obstante, existe un estudio científico público (tautológico, pero sin embargo...) es posible sobre los sentimientos, conductas, cogniciones, planes, motivos privados, etc. de una persona específica. El mensaje de la crítica es que ciertos elementos de la persona son estudiados y otros no y no necesariamente implica que otros elementos son el núcleo verdadero de la persona. La persona "toda" no puede ser estudiada de inmediato, así la limitación es legítima.

Además, se considera a la persona estable. Esto es empíricamente apoyado por medios estables y altos coeficientes de estabilidad de las cinco dimensiones (ver los impresionantes números de los coeficientes de estabilidad en: Costa \& McCrae, 1994, p.30 y 32). Las personas como un grupo y como individuos pueden cambiar, pero las cinco dimensiones no son constructos apropiados para descubrir el cambio (Helenos, 1993; Magia \& McFadden, 1994, p. 240) y estudiar a la persona en tanto el/ ella tenga rasgos individuales de diferencia.

También, la persona de las cinco dimensiones se describe con la ayuda de un número limitado de dimensiones generales y no con características específicas apropiadas para situaciones específicas. Con la ayuda de otros instrumentos de características específicas pueden evaluarse más adecuadamente (ver por ejemplo, Coolidge et al., 1994; que compara el DSMIII-R y las cinco dimensiones).

Igualmente, la persona se describe por medio del lenguaje común y la estructura se descubre al considerar sujetos de acuerdo a mediciones repetidas (ver por ejemplo, Ozer \& Gjerde, 1993). El lenguaje ordinario no es, de acuerdo a algunos científicos (por ejemplo, Mulaik, 1964) idealmente adecuado para la ciencia. Asimismo, la teoría implícita (estructura de las unidades) se considera a menudo determinada por los sesgos, el error lógico, las preocupaciones de los jueces (ver para una revisión 
equilibrada, por ejemplo, Semin, 1990; Semin \& Krahe, 1992). En defensa de estos comentarios puede afirmarse que ellos no disminuyen el hecho de que existe por lo menos en las ciencias sociales un traslapo entre el lenguaje cotidiano y el lenguaje científico. Son códigos diferentes con sus propias dinámicas. No pueden separarse completamente. Hasta el hallazgo más técnico tiene que ser explicado al público (el que paga los impuestos después de todo). Las dimensiones no son ficciones completamente linguísticas, pero existen niveles de ficción en el pensamiento humano. Este nivel tiene que analizarse y estimarse para las hipótesis léxica en búsqueda de la verdad (en el sentido de la correspondencia de afirmaciones y la realidad exterior) del enfoque.

Además de la reciente crítica hecha más arriba, siempre hubo deudas en los investigadores. La historia "revisionista" de Block (1995) sobre la investigación en las cinco dimensiones es apenas necesaria y puede ser considerada como un medio didáctico de exponer la crítica. La sugerencia de las palabras "historia revisionista" es que las faltas y errores del pasado continuarán siendo una carga en el presente. Este modelo psicodinámico de crecimiento de conocimiento es una hipótesis interesante. En nuestra opinión es más caprichoso. Algunos modelos de crecimiento admiten este capricho y son, hasta cierto punto, atractivos y bien elaborados. La investigación empírica, bien elaborada determinará su destino (Van Geert, 1991, 1994). A continuación, este sentido histórico de "culpa" será descrito.

La crítica empezó con Allport quien puede ser considerado como el padre y el crítico de las cinco dimensiones (John \& Robbins, 1993). Juzgó el estudio de las diferencias individuales como una de las metas de la psicología de la personalidad. "Comprender el caso individual y determinar las leyes del desarrollo del individuo son metas igualmente importantes y legítimas" (Allport, 1937, p. 303). Los modelos de estructura lineal para las diferencias individuales son caracterizadas como descriptivas, no la persona, pero orientada a la variable molar. "Una reducción factorial del vocabulario de personalidad es una bendición mixta; aquellos que buscan factores únicos considerarían el logro altamente significativo. Aquellos, por otro lado, que sostienen una teoría de rasgos que se yuxtaponen no lo harían" (Allport \& Odbert, 1936,pp. 31-32). Allport expresa parcialmente 
sus preferencias como psicólogo. Esto legitima, pero no lo haría, si los estudios específicos o perspectivas fueran requeridos como sólo "científicos" (o requeridos por el gobierno, por ejemplo, como sólo útiles).

Se reprocha a Cattell, a menudo, por su selección de las escalas 43 y 35. Esto no disminuye el hecho de que estos datos fueron sólo una parte de su base de datos para describir y explicar la personalidad. Una de las posibles razones por la cual Thurstone $\&$ Thurstone (1930) no descubrieron las dimensiones de personalidad sino las Habilidades Mentales Primarias fue que no esperaban mucho de las diferencias individuales para propósitos predictivos. Fiske (1949) fue uno de los primeros en abandonar los 16 factores de Cattell y describió las cinco dimensiones de diferencias individuales. No continuó esta clase de investigación porque consideraba las dimensiones como lingüísticas y no como fenómenos conductuales. Tupes \& Cristal (1961/1992, p. 247) consideraban como improbable que los cinco factores que habían encontrado serían los factores fundamentales de personalidad. Norman (1963) elaboró el trabajo de Allport y Odbert, pero fue crítico sobre las dimensiones, porque se derivaban del lenguaje. Esta crítica se repite desde el estudio de Allport y Odbert.

La búsqueda por dimensiones disposicionales continuó inclusive en un período encubierto, por la severa crítica del rasgo y de la teoría disposicional (Mischel, 1968). La interacción de persona y medio ambiente es obvia. La promesa de instrumentos nuevos y mejores no fue cumplida por esta perspectiva persona $\mathrm{x}$ medio ambiente. Los instrumentos más utilizados tomaron rasgo y disposición como conceptos. Wiggins \& Pincus (1992) caracterizaron la investigación de las cinco dimensiones como una tendencia de "vuelta a lo básico". Cada tendencia de vuelta a lo básico tiene ventajas y desventajas. Puede reorientar sobre preguntas pertinentes, pero corre también el riesgo de ser conservador, anticuado y hasta descuidando la ganancia de desarrollos posteriores.

Esta sección muestra que diversos componentes de las cinco dimensiones son críticamente examinados: número, contenido, estructura, validez de constructo y el poder predictivo, el poder explicativo y la generalidad. La persona que describe las cinco dimensiones es limitada. Esto último no es tanto una crítica como una evaluación. las dimensiones se 
determinan por un grupo representativo y por consiguiente los constructos describen sus características o estima parámetros de una población. No describen el desarrollo de un individuo y no son sensibles a éste. Block (1995) y Loevinger $(1993,1994)$ tienen razón al proponer que la dinámica de una persona no es cubierta por las cinco dimensiones, ya que se limitan a la descripción de un extraño y asumen estabilidad de las dimensiones. Descansan parcialmente en el lenguaje cotidiano falible y no revelan estructuras "genotípicas" o "profundas". Las dimensiones son generales y no pueden sustituir constructos e instrumentos específicos. Todas estas críticas requieren discusión de la perspectiva de las cinco dimensiones con otras perspectivas que reclaman cubrir los mencionados aspectos.

El desarrollo en la misma estructura de las cinco dimensiones, como una empresa científica es interesante porque $n i$ los investigadores ni los teóricos cambiarán las unidades (el léxico) y la estructura, sino la gente común porque sus respuestas están organizadas por el analista (factorial) de datos, quien actúa como su vocero (Hofstee, 1994).

El concepto de la persona de las cinco dimensiones encaja bien en el enfoque clásico disposicional, proveniente de Galton. Esta es la fuerza y limitación. Las personas tienen disposiciones (esencias). Otros conceptos de persona, por ejemplo, el existencial (por ejemplo, Sartre, 1965), lo fenomenológico y los conceptos postmodernos no son cubiertos por el enfoque de las cinco grandes dimensiones para la personalidad. Sin embargo, el enfoque si pretende hacer eso y por consiguiente no es culpable. Los otros enfoques tiene su propio trabajo conceptual y empírico y. son entonces capaces de confrontar su concepto y los hallazgos con la "persona de las cinco dimensiones".

\section{Conclusiones y Comentarios}

Las cinco dimensiones son generales e independientes para diferenciar entre adultos. Existe consenso sobre el espacio del lenguaje de la personalidad. Hecho que durará firmemente, por un tiempo. El contenido tiene que ser refinado comparando las cinco dimensiones con otras escalas disposicionales de diferencias de personalidad. En esta comparación tiene que prestarse más atención a las diferencias de éstas con otras escalas de personalidad y constructos relacionados. Se recomienda estudios funcio- 
nales, en los cuales el uso de las cinco dimensiones se estudia para grupos específicos (padres, maestros, pares, grupos de edad, profesionales, como gerentes de personal, enfermeras, doctores en medicina, psiquíatras) y situaciones específicas (por ejemplo, conversacional, profesional, neutral y selección). La experiencia y la investigación con el esquema "persona $\mathbf{x}$ situación" ha ofrecido la experiencia, los métodos y el conocimiento específico sobre como las dimensiones de personalidad en este tipo de estudios pueden salvarse de un determinismo ambiental puro (ver por ejemplo, Bem \& Allen, 1974; Epstein, 1979, 1980).

Al predecir las conductas pertinentes, los resultados de las cinco dimensiones son modestos, lo que difiere de resultados de otras escalas de personalidad. La generalidad del logro predictivo de las cinco dimensiones es aun inferior (no obstante significativo) que el de los instrumentos mas especializados (por ejemplo, las Escalas de Hogan). Se encuentran algunas relaciones interesantes con las cinco dimensiones como un instrumento predictivo, pero para decisiones individuales, éstas tienen sus limitaciones por lo que se espera mejore. Las cinco dimensiones explican sólo una pequeña parte de la varianza, independientemente de las dimensiones cognitivas. Para mejorar la predicción de los criterios pertinentes, el psicólogo tiene que encontrar otros modos.

El estatus teórico de la discusión de las cinco dimensiones tiene que continuar o quizás iniciarse a un nivel fundamental. Esta cuestión no puede ser resuelta únicamente por la investigación empírica, implica, entre otros, el análisis teórico de la relación entre el lenguaje ordinario y el de las ciencias sociales (Fletcher, 1993). Existen niveles de estudio de la personalidad psicológica: un nivel léxico que estudia los constructos implícitos de personas (en el sentido abstracto), el nivel de las redes nomológicas de constructos y un nivel de modelado (matemático) de algunas conductas claramente circunscritas. También se incluye la cuestión de si la teoría es necesaria en la psicología de la personalidad o si el análisis de las estructuras implícitas de grupos representativos será suficiente (Brokken, 1978; Hofstee, 1994). La preferencia por un nivel es prácticamente aceptable (la investigación debe continuar) pero teóricamente no justificado. Tal como se diría, la verdad no la posee una sola operacionalización (Hofstee, 1994), se puede decir que el conocimiento de la personalidad no lo posee sola- 
mente el lenguaje ordinario, la teoría psicodinámica, disposicional o los modelos matemáticos. La meráfora del procesamiento paralelo puede ayudar para comprender la perspectiva de la persona (personalidad), de los conceptos y de teorías que tienen que funcionar de modo paralelo. Esto lleva a elegir el "antieclectismo" (el dictum Hegeliano: "Das Wahre ist das Ganze", es decir, la verdad que es un todo) y el "antiselectivismo" (es decir, en contra del único constructo central). Esto permite, por supuesto, el estudio sobre niveles específicos, en la medida que no pueden hacerse estudios que toquen todos los niveles de una vez, pero esto no implica que uno pueda actuar como si hubiera un solo nivel. Pervin (1994) ha indicado los diferentes niveles legítimos que pueden estudiarse para llegar a comprender la persona. Las cinco dimensiones son todavía no teóricas y serán un reto para conectar las dimensiones con diferentes teorías y niveles. La discusión con respecto a las pruebas de inteligencia puede servir como ejemplo, en la medida que las más utilizadas no fueron desarrolladas bajo la guía de una teoría. Muchos análisis correlacionales y conceptuales elucidan factores de inteligencia. Por ejemplo, Demetriou, Efklides \& Plasidou (1993) argumentaron, de la siguiente manera, la existencia de diferentes factores de inteligencia: La mente humana está organizada para simbolizar, contar, hacer arreglos espaciales, etc., y el mundo físico permite ordenar los aspectos centrales de aquella manera. Este es un ejemplo de la transición de un nivel a otro o de la conexión de dos niveles que es el comienzo del procesamiento paralelo.

Wiggins \& Pincus (1992) argumentaron que los estudios de la cinco dimensiones representan un movimiento de "regreso a lo básico" en el estudio de la personalidad. Esto se puede apreciar si se retira lo irrelevante del estudio de la personalidad, limitándose cada enfoque. Pero ¿se retira lo irrelevante? Las cinco dimensiones no están conectadas con contextos y no son sensibles para la dinámica o desarrollo y tampoco sobre lo que nos gustaría saber más, nosotros mismos y nuestras intimidades, pero para saber sobre un extraño. La teoría clásica disposicional se aproxima a las cinco dimensiones, ésta no es relacional ni contiene, por lo menos, un intento de ser "transcontextual" y "transituacional". Emde (1994, p. 719) escribe que, como psiquiatra infantil, había aprendido tres cosas importantes: "Conócete a ti mismo" (No: conocer a un extraño), "nada en exceso" (la famosa virtud aristoteliana) y "saber que lo que uno conoce 
es relacional". Las cinco dimensiones existen como una estructura constituida por un referéndum y analizadas por el analista factorial quien $\mathrm{da}$, como un demócrata, a cada respuesta, el mismo peso. La estructura no es una estructura de una persona específica. La investigación sobre las cinco dimensiones ha ofrecido la estructura y el contenido de nuestro lenguaje diario muy claramente. Este lenguaje se muestra como inmanente y ampliamente conectado. Esta investigación y la taxonomía deja, no obstante, para bien o para mal muchas preguntas interesantes a la psicología de "la persona". Block (1982, 1995, p. 210). Menciona esta pregunta tan interesante y admite que hace su sugerencia más ambiciosa al pedir que sitúen las cinco dimensiones, constructos o como quieran ser llamados, de personalidad; "dentro de una estructura teórica intraindividual coherente (cursivas de Block). Este argumento también es expuesto por Magai \& McFadden (1995) y por Lewis (1995), quienes formulan conceptos que pueden ser útiles al estudiar la dinámica de una persona. Sin embargo, el trabajo empírico no ha empezado de un modo consistente. La investigación acerca de las cinco dimensiones indica que es hora de empezar la búsqueda por la persona.

\section{Referencias}

Allport, G.W. \& Odbert, H.S. (1936). "Trait-names: a psycho lexical study". Psychological Monographs, 47, No 211.

Angleitner, A., Ostendorf, F. \& John, O.P. (1990). Towards a taxonomy of personality descriptors in German: A psycho-lexical study. European Journal of Personality, 4.

Barrick, M.R. \& Mount, M.K. (1991). The Big Five personality dimensions and job performance. Personnel Psychology, 44, 1-25.

Baumgarten, F. (1933). Die Charaktereigenschaften. In: Beitrage zur Charakter- und Personlichkeitsforschung. (Whole No 1) Berna: A. Francke.

Bem, D.J. \& Allen, A. (1974). On predicting some of the people some of the time: The search for cross-situational consistencies in behavior. Psychological Review, 81, 674-670.

Block, J. (1982). Assimilation, Accommodation, and the dynamics of personality development. Child Development, 53, 281-295. 
Block, J. (1995). A contrarian view of the Five-Factor-Approach to Personality Description. Psychological Bulletin, 117, 187-215.

Block, J. (1995). Going beyond the five factors given: Rejoinder to Costa and McCrae (1995) and Goldberg and Saucier (1995). Psychological Bulletin, 117, 226-229.

Block, J.H. \& Block, J. (1980). The role of ego-control and ego-resilience in the organization of behavior. In: W.A. Collins (Ed.), The Minnesota Symposia on Child Psychology. Vol. 13. (pp. 39-101). Hillsdale NJ: Erlbaum.

Briggs, S.R. (1992) Assessing the Five-Factor Model of personality description. Journal of Personality, 60, 253-293.

Brokken, F.B. (1978). The language of personality. Unpublished doctoral dissertation, Universidad de Groningen, The Netherlands.

Brokken, F.B. \& Smid, N.G. (1984), Een uitbreiding van de Standaard Persoonlijkheids Eigenschappen Lijst (SPEL). (An extension of the Standard Personality-Characteristics-List). Nederlands Tijdschrift voor de Psychologie, 39, 348-352.

Bruner, J.S. \& Tagiuri, R. (1954). The perception of people. In: G. Lindzey (Ed.), Handbook of Social Psychology (Vol. 2). Reading MA: Addison-Wesley.

Campbell, D.T. \& Fiske, D.W. (1959). Convergent and discriminant validity by the multitrait multimethod matrix. Psychological Bulletin, 56, 81-105.

Cattell, R.B. (1943). The description of personality: the foundations of trait measurements. Psychological Review, 50, 476-507.

Cattell, R.B. (1995). The fallacy of the five factors in the personality sphere. The Psychologist, May 1995. pp. 207-208.

Church, A.T. (1994). Relating the Tellegen and the Five-Factor Model of personality structure. Journal of Personality and Social Psychology, 67, 898-909.

Clancy Dollinger, S.M. (1995). Identity styles and the Five-Factor Model of Personality. Journal of Research in Personality, 29, 475-479.

Clancy, S.M. \& Dollinger, S.J. (1993). Identity, self, and personality" I. Identity status and the Five-Factor Model of personality. Journal of Research on Adolescence, 3, 227-245.

Coolidge, F.L. Becker, L.A., DiRito, D.C., Durham, R.L. Kinlaw, M.M. \& Philbrick, P.B. (1994). On the relationship of the Five-Factor 
personality model to personality disorders: Four reservations. Psychological Reports, 75, 11-21.

Costa, P.T. \& McCrae, R.R. (1985). The NEO Personality Inventory Manual. Odessa, FL: Psychological Assessment Resources.

Costa, P.T. \& McCrae, R.R. (1992), Multiple use for longitudinal personality data. European Journal of Personality, 6, 85-102.

Costa, P.T. \& McCrae, R.R. (1992). Normal personality assessment in clinical practice. Psychological Assessment, 4, 5-13.

Costa, P.T. \& McCrae, R.R. (1992). The Five-Factor Model of personality and its relevance to personality disorders. Journal of Personality Disorders, 6, 343-359.

Costa, P.T. \& McCrae, R.R. (1994). Set like plaster? Evidence for the stability of adult personality. In: T.F. Heatherton and J.L. Weinberger, Can Personality Change? Washington, DC: American Psychological Association.

Costa, P.T.\& McCrae, R.R. (1995). Solid grounds in the wetlands of Personality: A Reply to Block. Psychological Bulletin, 117, 216-220. Cronbach, L.J. (1955). Processes affecting scores on "understanding others" and assumed similarity. Psychological Bulletin, 52, 177-179.

Dam, K. van (1996). Dansende beren; beoordelingsprocessen bij personeelsselectie. (Dancing bears: judgment processes in personnel selection). Enschede: Copyprint 2000.

Demetriou, A., Efklides, A. \& Platsidou, M. (1993). The architecture and dynamics of the developing mind. Monographs of the Society for Research in Child Development, 58, 5-5- serial No 234.

De Raad, B. (1992). The replicability of the Big Five personality dimensions in three word-classes of Dutch language. European Journal of Personality, 6, 15-29.

De Raad, B. (1994). An expedition in search of a fifth universal factor: key issues in the lexical approach. European Journal of Personality, 8, $1-22$.

De Raad, B. (1996, in press). The Big Five; History, procedures, developments, and applications. De Raad, B. \& Van Heck, G.L. (Eds.), Special issue: European Journal of Psychology. Vol. 8.

De Raad, B., Hendriks, A.A.J. \& Hofstee, W.K.B. (1992). Towards a refined structure of personality traits. European Journal of Psychology, 6, 301-319. 
De Raad, \& Hofstee, W.K.B. (1993). A circumplex approach to the five factor model: A facet structure of trait adjectives supplemented by trait verbs. Person. Individ. Diff., 15, 493-505.

Digman, J.M. (1990). Personality structure: Emergence of the Five-Factor model. Annual Review of Psychlogy, 41, 417-440.

Digman, J.M. \& Takamoto-Chock, N.K. (1981). Factors in the natural language of personality: re-analysis, comparison, and interpretation of six major studies. Multivariate Behavioral Research, 16, 149-170.

Emde, R.N. (1994). Individuality, Context, and the Search for meaning. Child Development, 65, 719-737.

Epstein, S. (1979). The stability of behavior: I. On predicting some people most of the time. Journal of Personality and Social Psychology, 37, 10971126.

Epstein, S. (1980). The stability of behavior: II. Implications for psychological research. American Psychologist, 35, 790-806.

Fiske, D.W. (1949). Consistency of the factorial structures of personality ratings form different sources. Journal of Abnormal and Social Psychology, 44, 329-344.

Fletcher, G.J.O. (1993). The scientific credibility of commonsense psychology. In: K.H. Craik, R. Hogan \& R.N. Wolfe, Fifty years of personality psychology. Nueva York: Plenum Press.

Foa, U.G. (1965). New developments in facet analysis and design. Psychological Review, 72, 262-274.

Funder, D.C. (1987). Errors and mistakes: Evaluating the accuracy of social judgment. Psychological Bulletin, 101, 75-90.

Funder, D.C. (1989). Accuracy in personality judgment and the dancing bear. In: D.M. Buss \& N. Cantor (Eds.), Personality psychology: Recent trends and emerging directions. (pp. 210-223). Nueva York: Springer. Fund̦er, D.C. (1993). Judgments of personality and personality itself. In:

K.H. Craik, R. Hogan \& R.N. Wolfe, Fiffy years of personality psychology.

Nueva York: Plenum Press.

Galton, F. (1883). Inquiries into human faculties and its development. Londres: MacMillan.

Galton, F. (1884). "Measurement of character". Fortnightly Review, 36, 179-185.

Geert, P. van (1991). A dynamic systems model of cognitive and language growth. Psychological Review, 98, 3-53. 
Geert, P. van (1994). Dynamic systems of development, Change between complexity and Chaos. Nueva York: Harvester Wheatsheaf.

Gottfredsen, G.D., Jones, E.M. \& Holland, J.L. (1993). Personality and Vocational Interests: The relation of Holland's six interest dimensions to five robust dimensions of personality. Journal of Consulting Psychology, 40, 518-524.

Goldberg, L.R. (1981). Language and individual differences: the search for universals in personality lexicons. In: L. Wheeler (Ed.). Review of Personality and Social Psychology, Vol. 2, pp. 141-165. Sage: Beverly Hills, CA.

Goldberg, L.R. (1990). An alternative "description od personality": The Big Five factor structure. Journal of Personality and Social Psychology, 59, 1216-1229.

Goldberg, L.R. (1995). What the hell took so long? Donald W. Fiske and the Big-Five Factor Structure. In: P.E. Shrout and S.T. Fiske: Personality Research, Methods, and Theory. A Festschrift honoring Donald W. Fiske. Hillsdale, NJ: Erlbaum.

Goldberg, L.R. \& Saucier, G. (1995). So what do you propose we do instead? A reply to Block. Psychological Bulletin, 117, 221-225.

Goedhart, A.W., Treffers, Ph. D.A. \& Kohnstamm, G.A. Het vijf-factoren model in de kinderpsychiatrie: Vrije persoonsbeschrijvingen door ouders van hun kinderen. (The Big Five model in child psychiatry: Free personality descriptions by parents of their children). Pedagogische Studien, 69, 40-50.

Gough, H.G. (1987). California Psychological Inventory: Administrator's guide. Palo Alto CA: Consulting Psychologists Press.

Habermas, J. (1979). Communication and Evolution of Society. Boston: Beacon Press.

Havill, V., Halverson, C.F. \& Kohnstamm, G.A. (1994). Parents' use of Bif Five categories in their natural language descriptions of children. In: C.F. Halverson, G.A. Kohnstamm and R.P. Martin (Eds.), The developing Structure of Temperament and Personality from Infancy to Adulthood. Hillsdale NJ: Erlbaum.

Heaven, P.C.L. (1996). Personality and self-reported delinquency: Analysis of the "Big Five" personality dimensions. Pers. Individ. Diff., 20, 4754. 
Helson, R. (1993). Comparing studies of adult development: Toward a paradigm of tension between stabilty and change. In: D.C. Funder, R.D. Parke, C. Tomlinson-Keasy and K, Widaman, Studying lives through time. Washington, DC: American Psychological Association. Hendriks, A.A.J., Hofstee, W.K.B., De Raad, B. \& Angleitner, A. (november 1995). The Five-Factor Personality Inventory (FFPI). Not published brief report, Universidad de Groningen, The Netherlands, Department of Psychology.

Hofstee, W.K.B. (1994). Who should own the definition of personality? European Journal of Personality, 8, 149-162.

Hofstee, W.K.B., de Raad, B. \& Goldberg, L.R. (1992). Integration of the Big Five and circumplex approaches to trait structure. Journal of Personality and Social Psychology, 63, 146-163.

Hogan, R. (1986). Hogan Personality Inventory manual. Minneapolis MN:

National Computer Systems.

Hogarth, R.M. (1980; 2nd edition, 1987). Judgment and Choice. Manchester, Inglaterra: J. Wiley.

Jackson, D.N. (1984). Personality Research Form Manual (3rd edition) Port Huron, MI: Research psychologists press.

Jackson, D.N. Paunonen, S.V., Fraboni, M. \& Goflin, R.D. (1996). A five-factor version versus a six-factor model of personality structure. Person. Individ.Diff., 20, 33-45.

John, O.P. (1990).The Big Five taxonomy: Dimensions of personality in natural languages and in questionnaires. In: L. Pervin (Ed.), Handbook of Personality: Theory and Research. (pp. 66-100). Nueva York: Guilford Press.

John, O.P., Caspi, A., Robins, R.W. \& Stouthamer-Loeber, M. (1994). The "Little Five": Exploring the nomological network of the FiveFactor Model of Personality in adolescent boys. Child Development, 65, 160-178.

John, O.P. \& Robins, R.W. (1993). Gordon Allport, Father and Critic of the Five-Factyor model. In: Craik, K.H., Hogan, R. and R.N. Wolfe, Fifty years of Personality Psychology. Nueva York: Plenum Press. Klages, L. (1932). The Science of Character. London: George Allen \& Unwin.

Kohnstamm, G.A. (1992). Factoren in gedragsbeoordelingen van leerlingen. (Personality factors in teacher ratings of schoolchildren: Resemblance 
with the Big Five). Pedagogische Studien, 69, 12-22.

Kohnstamm, G.A., Halverson, Havill, V.L. \& Mervielde, I. (1993). Parents' free descriptions of child characteristics: A cross- cultural search for the roots of thew Big Five. In: S. Herkness \& C.M. Super (Eds.), Parents' cultural belief systems: Cultural origins and consequences. Nueva York: The Guilford Press.

Kluckhohn, C. \& Murray, H.A. (1953). Personality formation: The determinants. In: C. Kluckhohn and D.M. Schneider (Eds.), Personality in nature, society, and culture (pp. 53-67). Nueva York: Alfred A. Knopf.

Laak, J. ter (1994). Accuraatheid van het oordelen over persoonlijkheidseigenschappen en cognitieve ontwikkeling van leerlingen. (Accuracy of judgments of personality characteristics and cognitive capacities of pupils). Tijdschrift voor Ontwikkelingspsychologie, $21,39-51$.

Laak, Jan J.F. (1995). Psychologische Diagnostiek, inhoudelijke en methodologische grondslagen. (Psychological Diagnostics: Content and Method). Lisse: Swets \& Zeitlinger.

Lakatos, I. (1978). The methodology of scientific programmes. Cambridge Inglaterra: Cambridge University Press.

Lewis, M. D.(1995). Cognition-emotion feedback and the self-organization of developmental paths. Human Development, 38, 71-102.

Lieshout, C.F.M. \& Haselager, G.J.T. (1992). Persoonlijkheidsfactoren in

Q-Sort persoonsbeschrijvingen van kinderen: Realtie tot het vijf factoren model. (Personality dimensions in California Child Q-set descriptions: Relations with the Five Factor Model). Pedagogische Studien, 69, 2339.

Loehlin, J.C. (1992). Genes and environment in personality development. Newbury Park CA: Sage.

Loevinger, J. (1957). Objective tests as instruments of psychological theory. Psychological Reports, 3, 635-694.

Loevinger, J. Conformity and conscientiousness: One factor or two Stages? In: D. C. Funder, R.D. Parke, C Tomlinson-Keasy and K. Widaman. Studying Lives through time. Washington, DC: American Psychological Association.

Loevinger, J. (1994). Has psychology lost its conscience. Journal of Personality Assessment, 62, 2-8. 
MacKay, D.G. (1993). The theoretical epistemology: a new perspective on some long-standing methodological issues in psychology. In: G. Keren and C. Lewis, $A$ handbook od data nanalysis in the Behavioral Sciences: Methodological Issues. Hillsdale NJ: Erlbaum.

Magai, C. \& McFadden, S.H. (1995). The role of emotions in social and personality development. Nueva York: Plenum Press.

Marshall, G.N., Wortman, C.B., Vickers, R.R. Kusulas, J.W. \& Hervig, L.K. (1994). The Five-Factor Model of Personality as a framework for Personality-Health research. Journal of Personality and Social Psychology, 67, 278-286.

Mayer, J.D. (1995). A framework for the classification of personality components. Journal of Personality. 63, 819-878.

McAdams, D.P. (1992). The Five-Factor model in personality: A critical appraisal. Journal of Personality, 60, 329-361.

McAdams, D.P. (1994a). The Person. An introduction to personality psychology (Second Edition). Nueva York: Hartcourt Brace College Publishers.

McAdams, D.P. (1994b). Can Personality Change? Levels of stability and growth in personality across the lifespan. In: T. Heatherton and J. Weingberger (Eds.), Can Personality Change?. (pp. 299-315). Washington DC: American Psychological Association.

McCrae, R.R. \& Costa, P.T. \& Piedmont, R.L. (1993). Folk concepts, Natural Language, and Psychological Constructs: The California Psychological Inventory and the Five-Factor Model. Journal of Personality, 61, 1-26.

Messick, S. (1989). Validity. In: R.L. Linn (Ed.), Third Edition. Educational Measurement. National Council on Measurement in Education. Londres: Collier MacMillan Publishers.

Mischel, W. (1968). Personality and Assessment. Nueva York: J. Wiley \& Sons.

Mulaik. S.A. (1964). Are personality factors raters' conceptual factors? Journal of Consulting Psychology, 28, 506-511.

Narayanam, L. Menon, S. \& Levine, E.L. (1995). Personality structure:

A culture-specific examination of the five-factor model. Journal of Personality Assessment, 64, 51-62.

Nisbett, R.E. \& Ross, L.D. (1980). Human Inference: Strategies and shortcomings of social judgment, Englewood Cliffs NJ: Prentice Hall. 
Norman, W.T. (1963). Toward an adequate taxonomy of personality attributes: replicated factor structure in peer nomination personality ratings. Journal of Abnormal and Social Psychology, 66, 574-583.

Ostendorf,F. (1990). Sprache und Personlichkeitsstruktur: Zut Validitat des Funf-Faktoren Modells der Personlichkeit. Regensburg, Alemania: S. Roderer Verlag.

Ostendorf, F. \& Angleitner, A. (1993). A comparison of different instruments proposed to measure the Big Five. A contribution to the symposium: "Assessement through personality questionnaire". Second European Conference on Psychological Assessement, Groningen, The Netherlands, August 25-27, 1993.

Ozer, D.J. \& Gjerde, P.F. (1989). Patterns of personality consistency and change from childhood through adolescence. Journal of Personality and Social Psychology, 58, 472-486.

Passini, F.T. \& Norman, W.T. (1966). A universal conception of personality structure? Journal of Personality and Social Psychology, 4, 44-49.

Peabody, D. (1987). Selecting representative trait adjectives. Journal of Personality and Social Psychology, 52, 59-71.

Pepper, S.C. World hypotheses. Berkeley: University of California Press.

Pervin, L.A. (1994). Personality stability, personality change, and the questions of process. In: T.F. Heatherton and J.L. Weinberger, Can Personality Change? Washington DC: American Psychological Association.

Presley, R. \& Martin, R.P. (1994). Toward a structure of preschool temperament: Factor structure of the temperament Assessment battery for children. Journal of Personality, 62, 413-448.

Reese, H.W. \& Overton, W.F. (1970). Models of development and theories of development. In: P.B. Baltes \& L.R. Goulet (Eds.), Lifespan developmental psychology. Nueva York: Academic Press.

Revelle. W. (1995). Personality processes. Annual Review of Psychology, 46, 295-328.

Rumelin, G. (1890). Ueber die Temperamente. Deutsche Rundschau, 64, 397-412.

Sartre, J.P. (1965). Essays in Existentialism. Secaucus, Nueva Jersey: The Citadel Press.

Saucier, G. (1994). Mini-Markers: A brief version of Goldberg's Unipolar Bif-Five Markers. Journal of Personality Assessment, 63, 506-516. 
Semin, Gun R. (1990). Everyday assumptions, Language and Personality. In: C.R. Semin (Gun R.) \& K.J. Gergen. Everyday understanding: social and scientific implications. Londres: Sage Publications.

Semin, Gun R. \& Krahe, B. (1992). Personality language. In: G.V. Caprara and G.L. van Heck, Modern Personality Psychology, Critical reviews and new directions. Hertfordshire: Harvester Wheatsheaf.

Schmidt, F.L. \& Hunter, J.E. (1977). Development od a general solution to the problem of validity generalization. Journal of Applied Psychology, 62, 529-540.

Shaver, P.R. \& Brennan, K.A. (1992). Attachment styles and the "Big Five" personality traits: Their connections with each other and with romantic relations outcomes. Journal of Personality and Social Psychology Bulletin, 18, 536-545.

Shmelyov, A.G. \& Pokhil'ko, V.I. (1993). A taxonomy-oriented study of Russian personality-trait names. European Journal of Personality, 7, 117.

Snow, R.E. (1973). Theory construction for research on teaching. In:

R.M.W. Travers, Second Handbook for Research on Teaching. Chicago, Rand McNally.

Tellegen, A. (1982). Brief Manual for the Differential Personality Questionnaire. Unpublished manuscript, University of Minnesota. Thomae, H. (1990). How European is personality psychology in Europe. In: J.P.D. Drenth (Ed.). Psychology in Europe. (pp. 81-105). Amsterdam: North Holland Publishing Cy.

Thurstone, L.L. (1935). Vectors of Mind. Chicago: University of Chicago Press.

Thurstone, L.L. \& Thurstone, T.C. (1930). A neurotic inventory. Journal of Social Psychology, 1, 3-30.

Thurstone, L.L. \& Thurstone, T.C. (1941). Factorial studies of intelligence. Psychometric Monographs, Whole No 1 . Chicago: University of Chicago Press.

Tupes, E.C. \& Cristal, R.C. (1961). Recurrent personality factors based on trait ratings. (Tech. Rep. No ASD-TR-61-97). Lackland Air Force Base, Texas: U.S. Air Force.

Tupes, E.C. \& Cristal, R.C. (1992). Recurrent personality factors based on trait ratings. Journal of Personality, 60, 225-251. (Reprint of Tupes \& Cristal, 1961). 
Waller, N.G. \& Ben-Porath, Y. (1987). Is it time for clinical psychology to embrace th five-factor model of personality? American Psychologist, 42, 887-889.

Wiggins, J.S. (1968). Personality Structure. In: P.R. Fansworth (Ed.), Annual Review of Psychology, 19, (pp. 293-350). Palo Alto, California: Annual Reviews.

Wiggins, J.S. (1979). A psychological taxonomy of trait-desciptive terms: The interpersonal domain. Journal of Personality and Social Psychology, 37, 395-412.

Wiggins, J.S. \& Pincus, A.L. (1992). Personality: Structure and Assessment. Annual Review of Psychology, 43, 473-504. 